\title{
A metamodel for optimization problems
}

Ibrahim Assouroko

Peter Denno

This publication is available free of charge from: http://dx.doi.org/10.6028/NIST.IR.8096

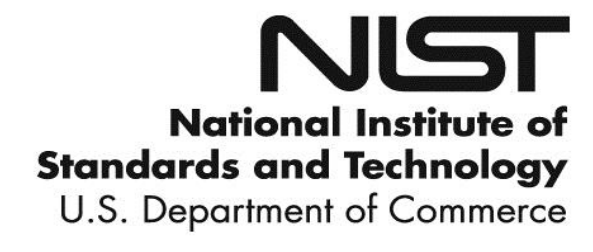




\title{
A metamodel for optimization problems
}

\author{
Ibrahim Assouroko \\ Peter Denno \\ Systems Integration Division \\ Engineering Laboratory
}

This publication is available free of charge from:

http://dx.doi.org/10.6028/NIST.IR.8096

January 2016

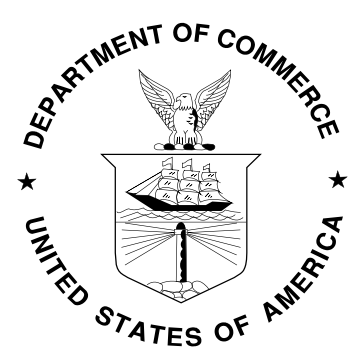

U.S. Department of Commerce

Penny Pritzker, Secretary

National Institute of Standards and Technology Willie May, Under Secretary of Commerce for Standards and Technology and Director 


\begin{abstract}
In mathematics, computer science, and operations research, mathematical optimization is the process of finding best feasible values (oftentimes maxima or minima) of a given objective that is modeled mathematically. This paper introduces the concept of a metamodel for optimization problems and describes an exploratory implementation of such a metamodel. The metamodel is (1) a conceptual model for capturing, in abstract terms, essential characteristics of a given optimization problem, and (2) a schema of sufficient formality to enable the problem modeled to be serialized to statements in a concrete optimization language. The exploratory metamodel presented in this paper addresses a majority of abstractions found in the OPL language. We present prototype software to compile OPL programs to populations of the metamodel and to serialize these populations back to OPL code. The paper describes the motivation for this work and a detailed specification of the exploratory implementation.
\end{abstract}

Keywords: metamodel, optimization problem, optimization model specification, Optimization Programming Language, UML diagram.

\title{
Contents
}

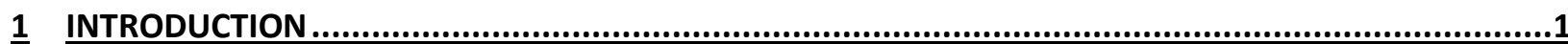

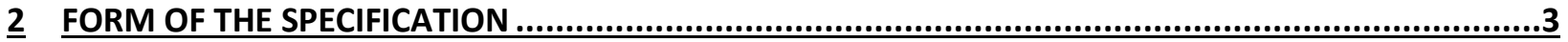

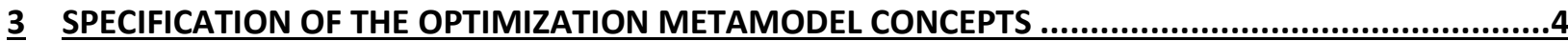

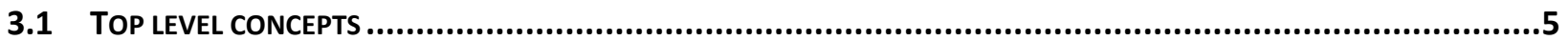

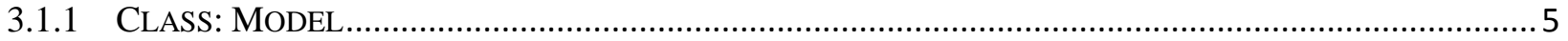

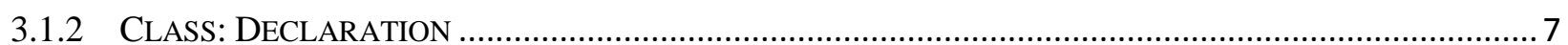

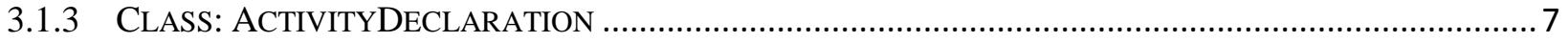

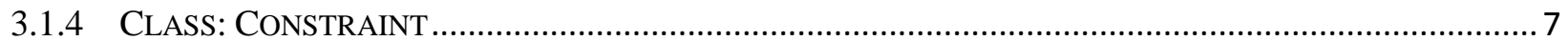

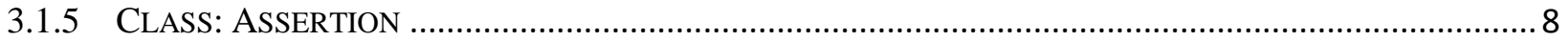

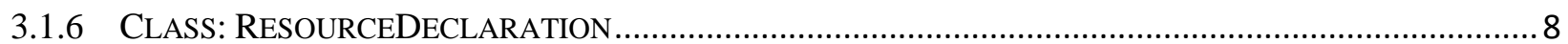

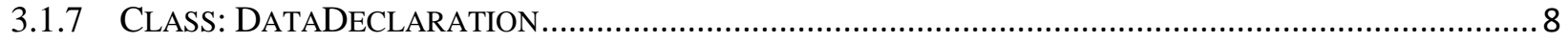

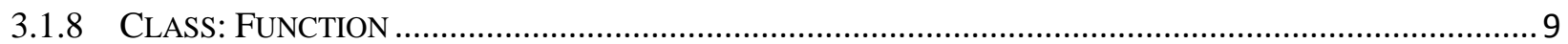

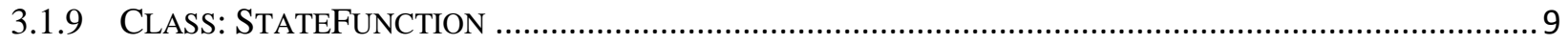

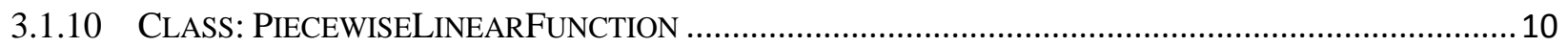

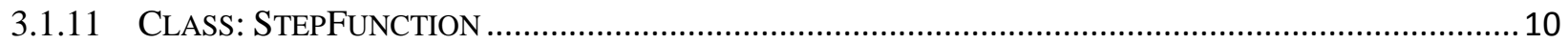

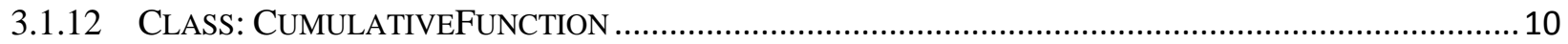

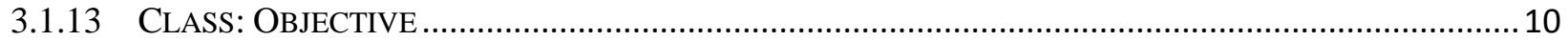

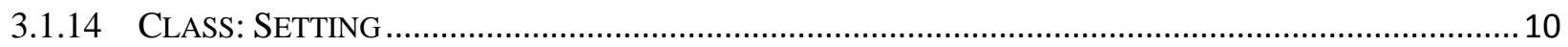

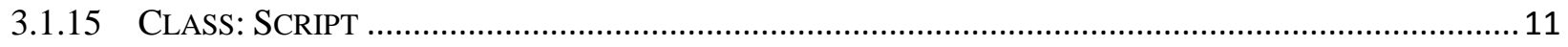

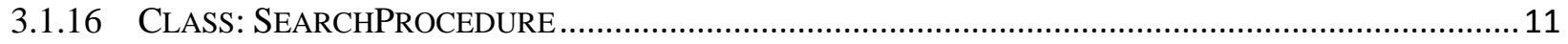

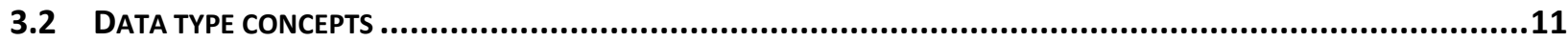

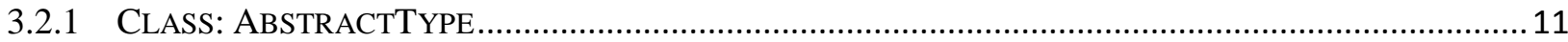

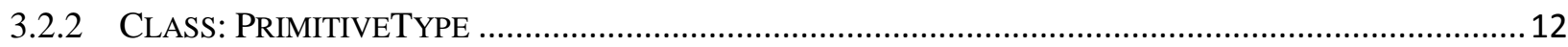

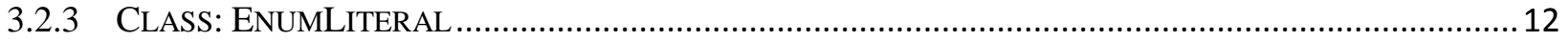




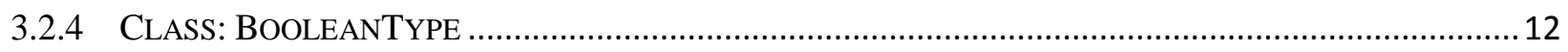

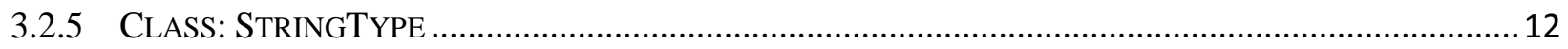

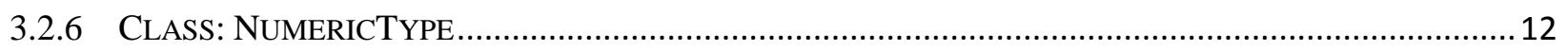

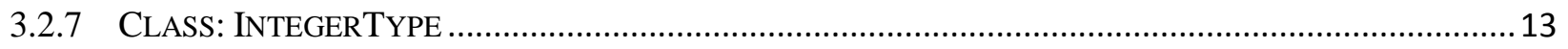

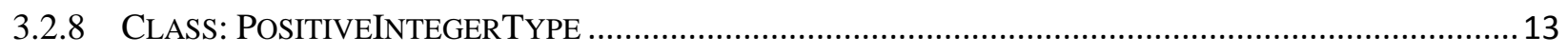

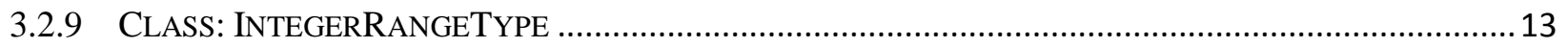

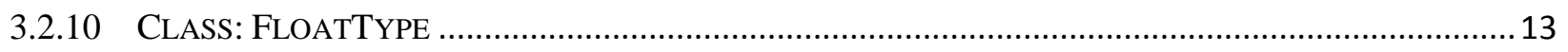

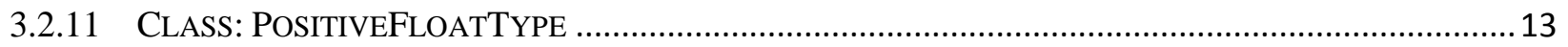

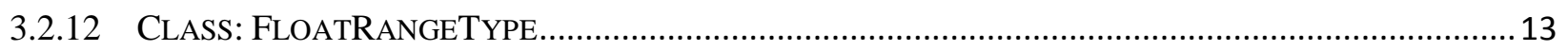

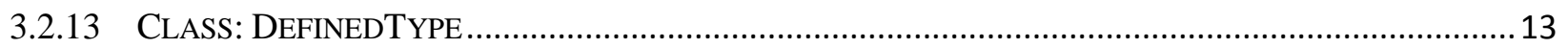

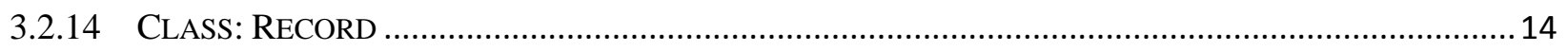

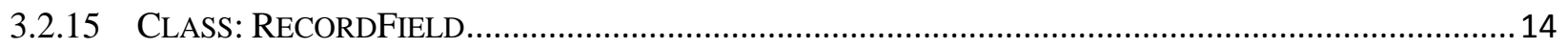

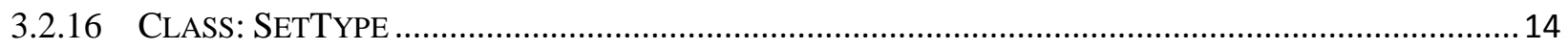

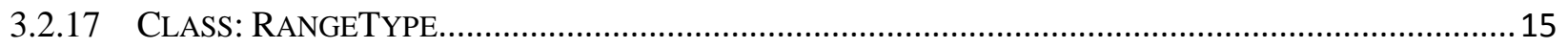

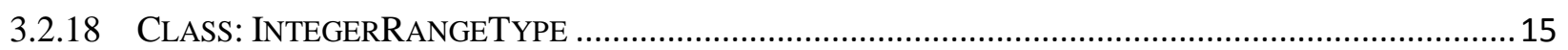

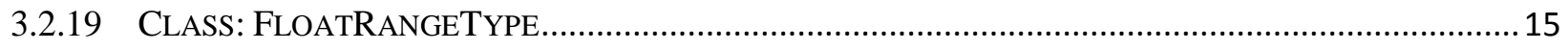

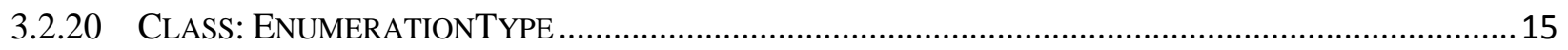

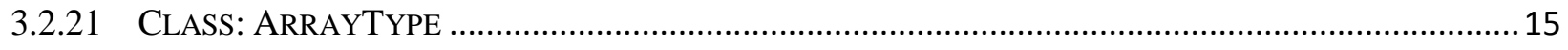

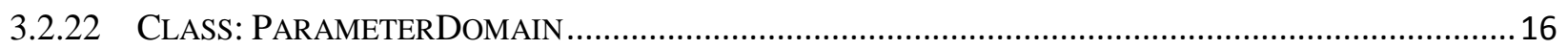

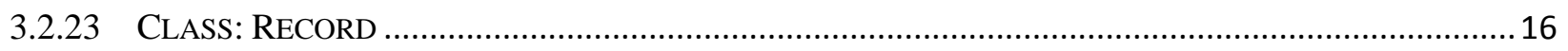

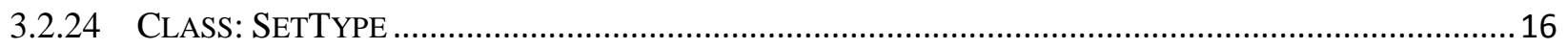

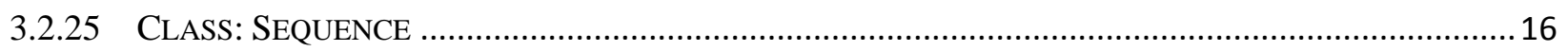

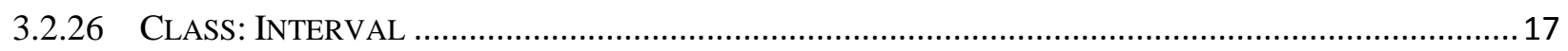

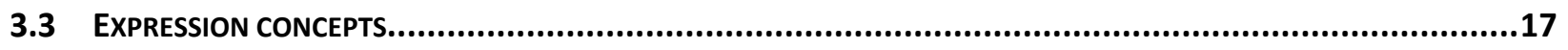

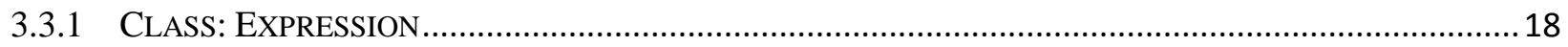

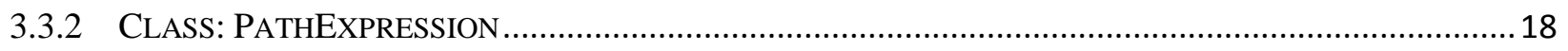

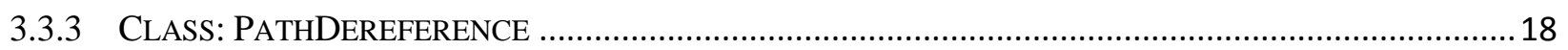

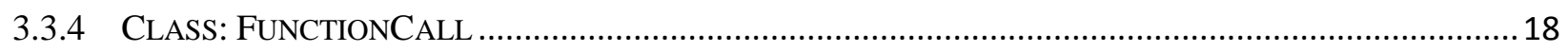

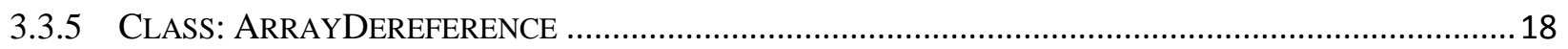

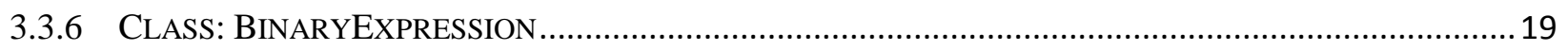

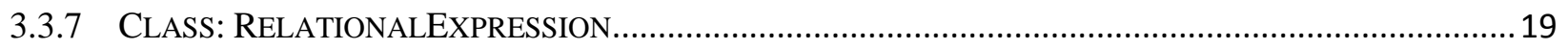

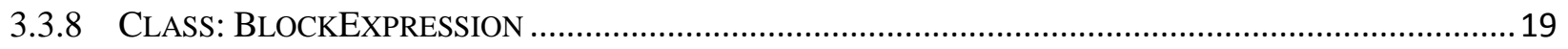

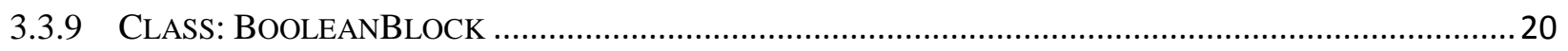

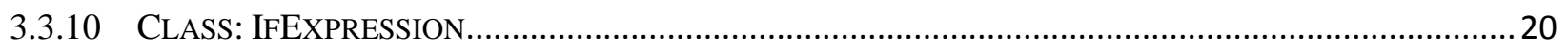

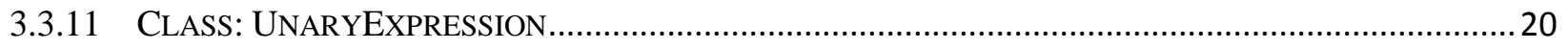

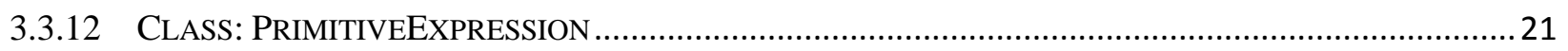

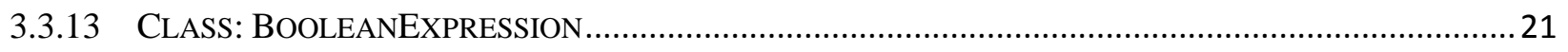

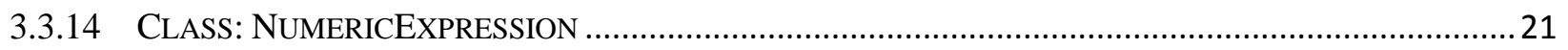

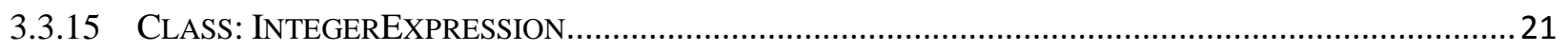

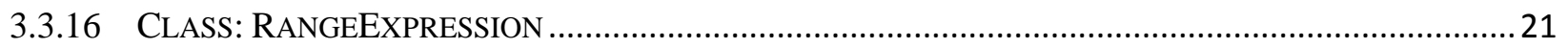

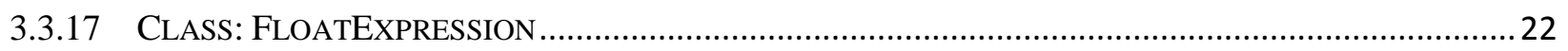

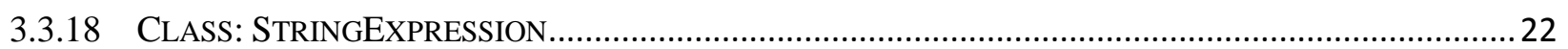




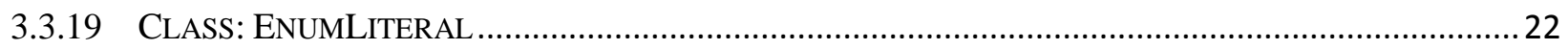

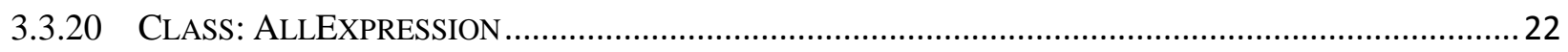

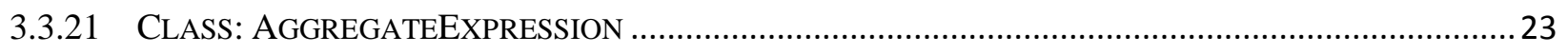

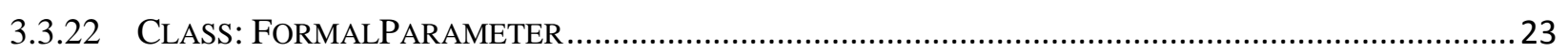

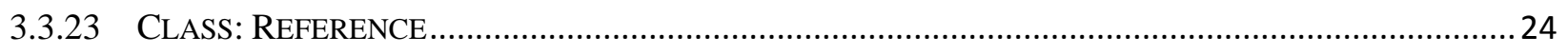

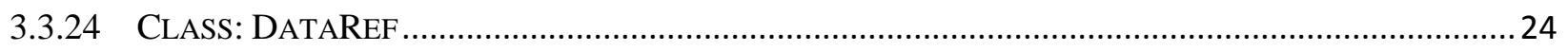

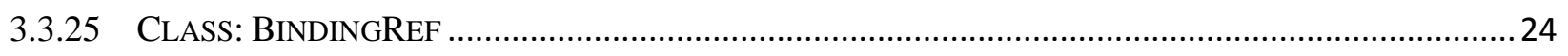

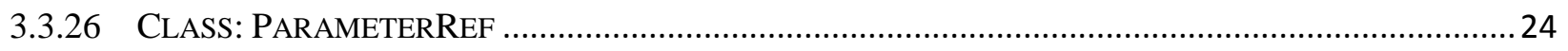

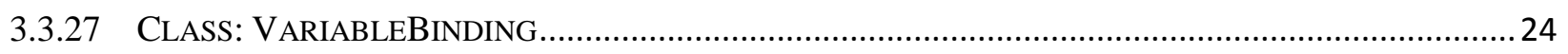

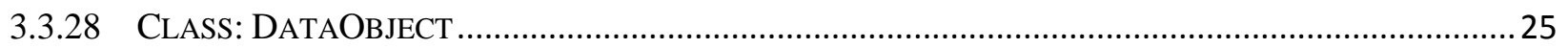

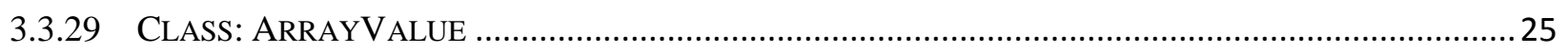

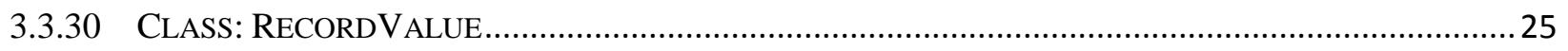

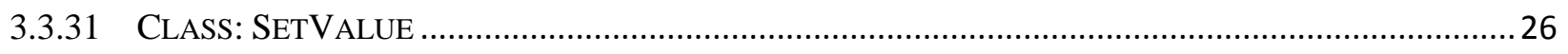

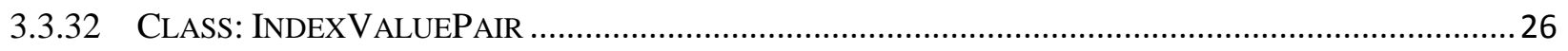

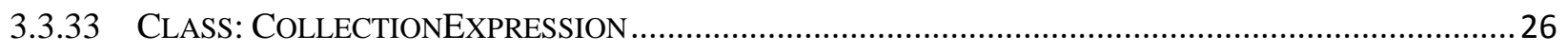

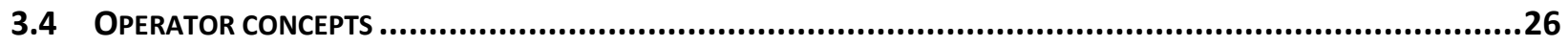

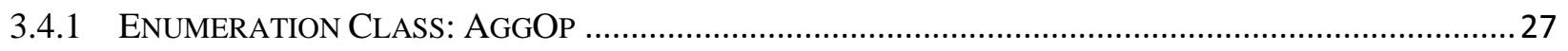

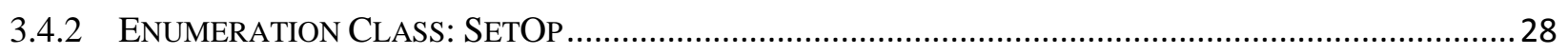

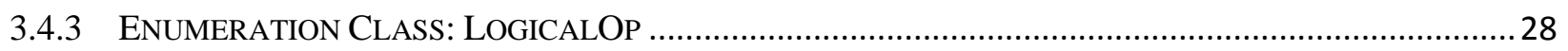

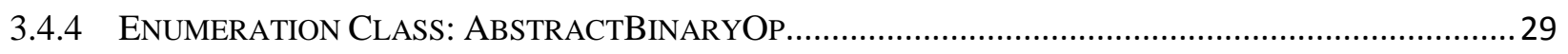

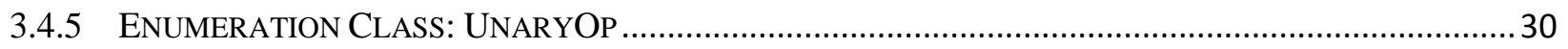

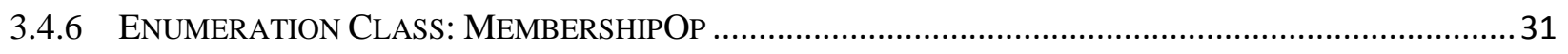

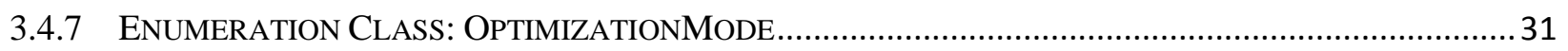

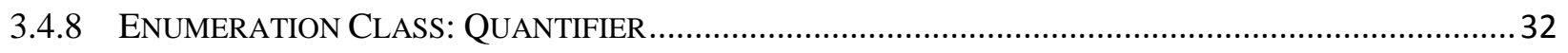

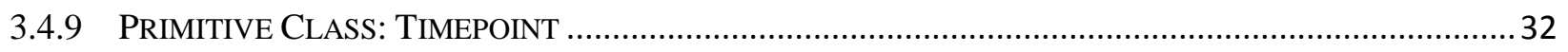

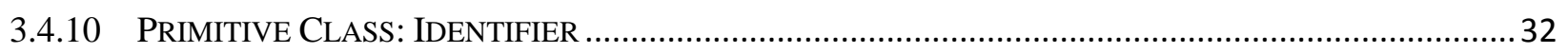

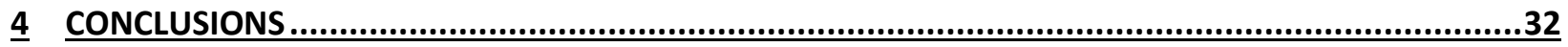

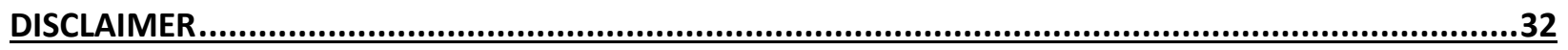

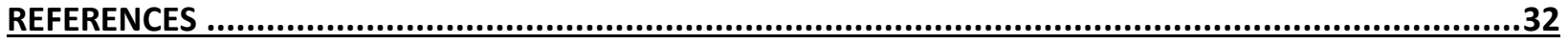

\section{Introduction}

This paper describes a metamodel of optimization problems. The sense of "metamodel" used here is consistent with usage of the term in information standards from the Object Management Group (OMG) including the Meta-Object Facility (MOF) [1] used to describe the Unified Modeling Language (UML) [2]]. Roughly speaking, in these standards, a metamodel is a conceptual model where the domain of discourse is some aspect of information technology. For example, for UML, the domain of discourse is objectoriented software; here, the UML metamodel provides for the description of things such as classes and 
methods. In this paper, the domain of discourse is optimization, of problems and software systems that solve those problems. The metamodel provides for the description of things such as objective functions and constraints. Ultimately, the metamodel should provide the breadth of detail necessary to serialize an instance optimization-metamodel to many algebraic modeling languages, such as OPL "Optimization Programing Language" []ㅡ, AMPL "A Mathematical Programing Language” [4], GAMS "General Algebraic Modeling System" [] [ 6] and AIMMS "Advanced Interactive Multidimensional Modeling System" [7]. Initially and in this paper we focus on the OPL language.

Metamodels provide capabilities beyond ordinary conceptual models. Specifically, a metamodel can serve as a storage form for models, and useful artifacts may be generated directly from model content. For example, instances of the UML metamodel can describe classes (model content) with sufficient fidelity that automated tools can generate Java class definitions from the class descriptions. These capabilities of metamodels are due to their more formal method of description.

Motivation for developing a metamodel of optimization problems are the resulting capabilities just described; the metamodel can serve as the storage form for optimization problems, from which can be generated actual, executable, optimization problems. The idea of a conceptual model for optimization problems is not new; languages such as AMPL [4] and GAMS [్] also provide such models along with mappings from the language to many executable tools (optimization code). An optimization metamodel provides the ability to programmatically develop optimization problems. This ability is important when using optimization technology in operational settings. For example, in manufacturing operations, information from production monitoring systems can be transformed to elements of an optimization problem using the metamodel. The metamodel instance can then be transformed to an executable optimization.

NIST is developing two tools to read OPL ${ }^{1}$ models into metamodel populations and to serialize those populations into OPL code. The former tool can be used to produce a "template" population that can be completed during problem formulation. (See Figure 1). The latter is used to produce an OPL model.

An example use of the metamodel in manufacturing is depicted in Figure 1. There a population conforming to the optimization metamodel is formulated from domain data and mapped into metamodel form, allowing serialization as an executable optimization. The top portion of the figure depicts a collection of manufacturing viewpoints relevant to some optimization problem such as finding optimal process parameters for a unit manufacturing process. Production information systems provide information in these viewpoints. The acquired information is used in problem formulation (middle third of Figure 1). Problem formulation concerns the development of design space constraints (typically mathematical inequalities) and objective function (typically an equation with weighted terms).

There are many possible technical means of formulating optimization problems. In this paper, we use engineering notebooks, an open notebook technology [8] integrated with semantic web technology [9] [10] [11]. The equations formulated by the user's work in the notebook are furnished to a partially completed metamodel population, thereby completing it. The completed metamodel population is then serialized into inputs to an optimization solver as depicted in the lower third.

\footnotetext{
${ }^{1}$ Optimization Programming Language (OPL) is an algebraic modeling language for mathematical optimization models. It is part of IBM's ILOG CPLEX Optimization Studio, an optimization software package.

(https://en.wikipedia.org/wiki/Optimization_Programming_Language)
} 


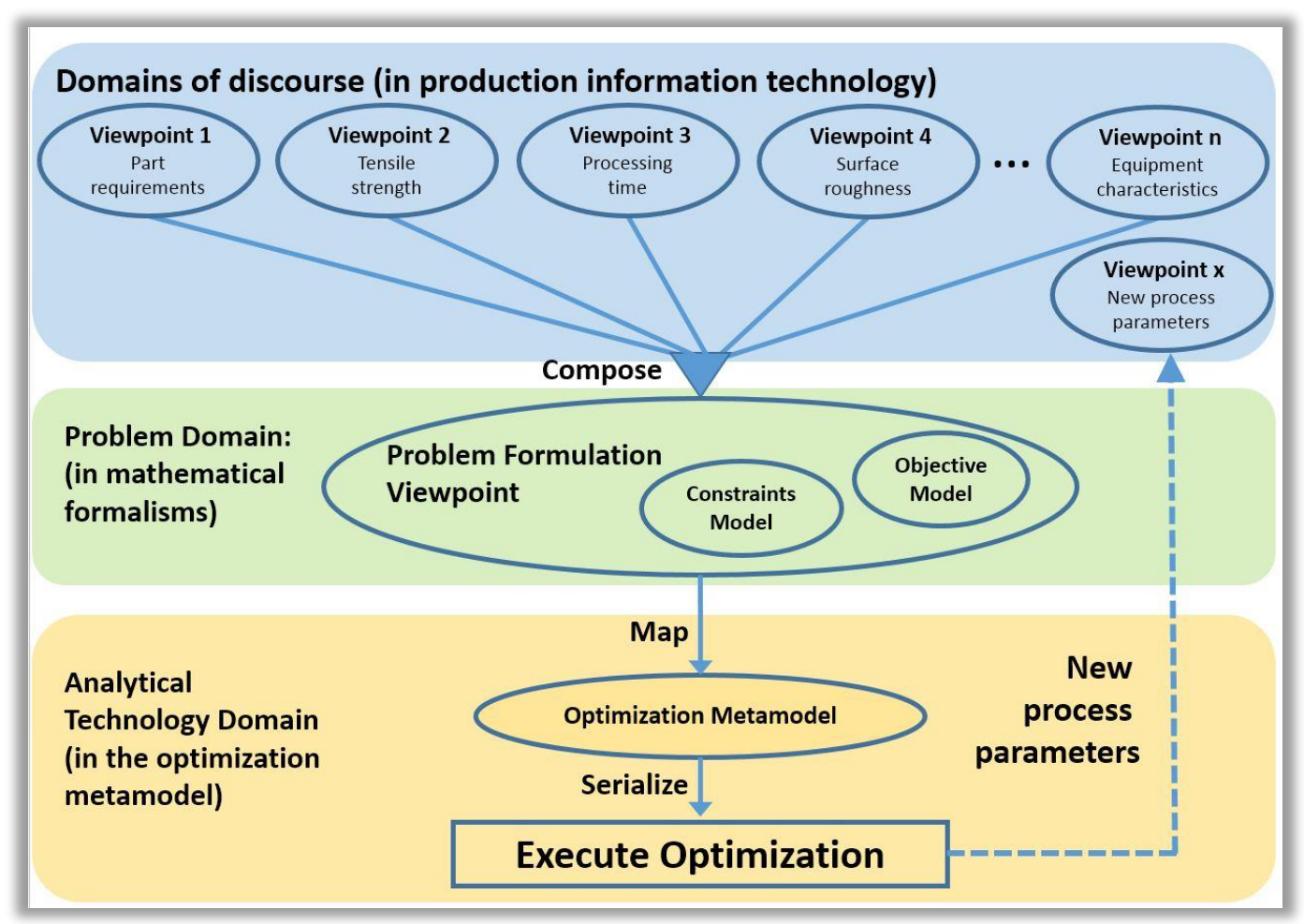

Figure 1: An example of using the metamodel in manufacturing.

The current metamodel can only address capabilities of OPL. NIST plans to extend the metamodel to address capabilities of other mathematical modeling languages. The objective in this paper is to begin to identify common elements of optimization problem specifications. The model specifications expressed in XMI "XML Metadata Interchange" standard can be found on the NIST GitHub site [12]. XMI is an OMG standard for exchanging metadata information via XML. It is commonly used as an interchange format for UML models, although it can also be used for serialization of metamodels.

The paper describes a specification of the proposed metamodel for optimization problems. Section 2 presents the form of the metamodel specification and the modeling technique used to make representation of the metamodel. Section 3 specifies the formal concepts in a set of UML diagrams. Each UML diagram represents a set of Classes corresponding to a set of optimization problem concepts.

\section{Form of the specification}

In this paper, metamodel elements are depicted as classes and associations in UML class diagrams. In UML, sets of concepts that are closely related are grouped into UML package constructs. Classes are depicted as boxes with partitions, and associations (called objectProperties) as labeled lines that connect the class boxes. Lines between class boxes with hollow arrow point on one end represent generalization/specialization relationships between the classes at the end of the line. Figure 2 shows an example UML diagram taken from the OptimizationTopLevelObjects UML package diagram. The upper partition of a class box provides the name of the class and sometimes special characteristics of the class 
itself. The lower partition of a class box shows the attributes (called dataProperties) associated with members of the class, if any. Figure 2 shows five classes: Function, StateFunction, PiecewiseLinearFunction, CumulativeFunction and StepFunction.

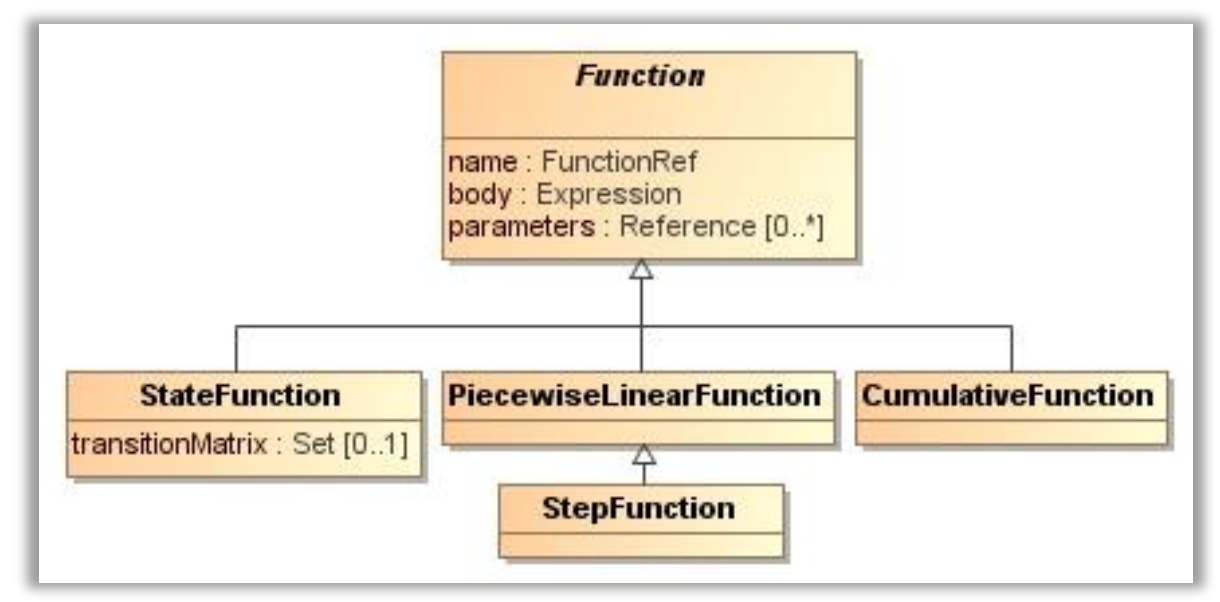

Figure 2: Example of UML classes arranged into a generalization or specialization hierarchy. Hollow arrows on the lines point to the more general class.

The sets of concepts represented in the metamodel are depicted as UML package diagrams in the form of classifications of concepts showing the hierarchy existing between those concepts. Some concepts are shown in more than one diagram to make it easier to understand dependencies between concepts not belonging to the same package. DataProperties are said to have multiplicities called cardinality constraints in UML that appear as numbers next to the property. The individual cardinality expressions are interpreted as follows:

- The cardinality "1" means "exactly one"

- The cardinality " $1 . . . *$ " means "at least one"

- The cardinality " $0 \ldots 1$ " means "at most one"

- The multiplicity " $0 \ldots$..." means "any number, zero or more"

In Figure 2, each "unnamed arrow" with open "head" between Function and StateFunction, PiecewiseLinearFunction, CumulativeFunction is called a generalization in UML. This means that StateFunction, PiecewiseLinearFunction, CumulativeFunction are sub classes of Function. In other words, every StateFunction, PiecewiseLinearFunction or CumulativeFunction is a Function, has a name, a body and may have zero or more parameters. The full model can be found on the NIST GitHub site.

\section{Specification of the optimization metamodel concepts}

This section of the paper describes the modeling of common concepts of the OPL language. As noted, these concepts are organized into UML package diagrams. Each model element is associated with one of the four packages defined in the metamodel: OptimizationTopLevel, OptimizationDataTypes, OptimizationExpressions, or OptimizationOperators. The main element in the OptimizationTopLevel package is the class Model. The class Model describes the optimization problem and serves as a container for the declaration of objective functions, constraints, and data types. The OptimizationExpressions package 
contains specifications of the optimization problem formulation through objective functions and constraints. OptimizationDataTypes contains specifications of the different data types used in an optimization problem. Common operators (See section 3.4) used in optimization problem formulation and data declaration are specified in OptimizationOperators package.

\subsection{Top level concepts}

The OptimizationTopLevel package contains classes describing the most fundamental elements of an optimization problem. These classes are depicted in Figure 3, Figure 4 and Figure 5.

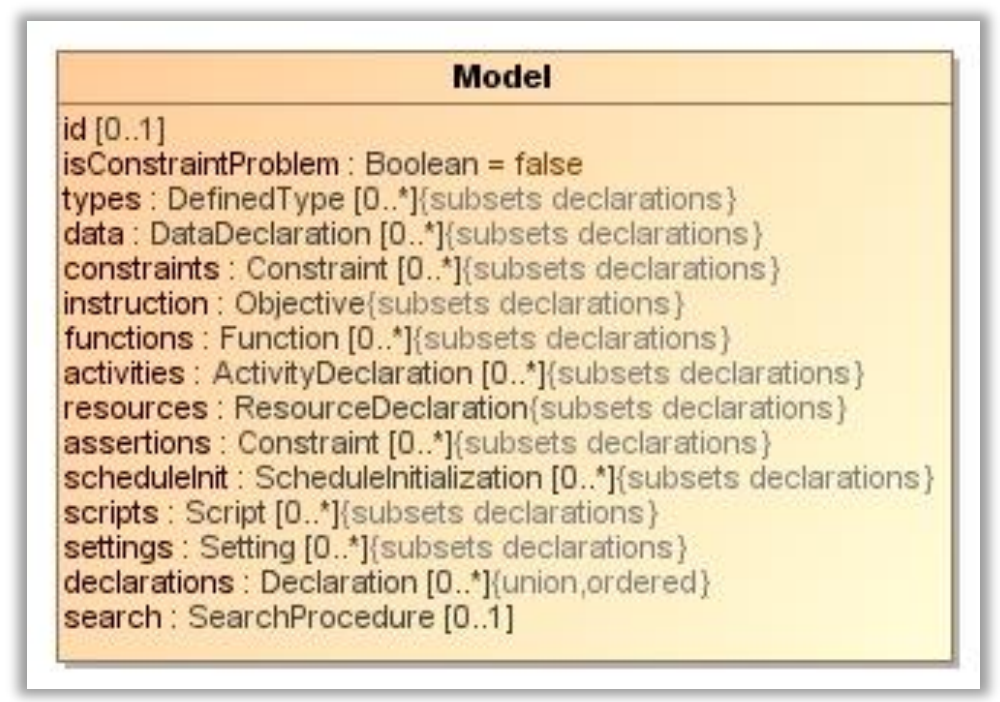

\section{Figure 3: Optimization model specification}

\subsubsection{Class: Model}

Definition: Model is an element that describes the optimization problem or a container for a complete optimization problem. A model consists of a set of declarations, such as data declaration, activity declaration, resource declaration, and can define, among other attributes, such attributes as types, data, constraints, instructions for objective functions, activities and resources.

\section{Properties}

\begin{tabular}{|l|l|}
\hline Attribute: id & Type: identifier
\end{tabular}

Definition: the unique (optional) identification of the model.

Cardinality: at most one

\begin{tabular}{|l|l|}
\hline Attribute: isConstraintProblem & Type: Boolean \\
\hline
\end{tabular}

Definition: a Boolean indicating whether or not this is a constraint satisfaction problem.

\begin{tabular}{|l|l|}
\hline Attribute: types & Type: DefinedType \\
\hline
\end{tabular}

Definition: a collection of defined types used in the model.

Cardinality: any number, zero or more 


\begin{tabular}{|l|l|}
\hline Attribute: data & Type: DataDeclaration \\
\hline
\end{tabular}

Definition: a collection of data objects declared in the model.

Cardinality: any number, zero or more

\begin{tabular}{|l|l|}
\hline Attribute: constraints & Type: Constraint \\
\hline
\end{tabular}

Definition: a collection of constraints defining the limits of the value produced by the objective.

Cardinality: any number, zero or more

\begin{tabular}{|l|l|}
\hline Attribute: instruction & Type: Objective \\
\hline
\end{tabular}

Definition: the Objective of the model; OR an expression to be satisfied by performing the analysis defined from the model.

\section{Attribute: functions}

\section{Type: Function}

Definition: a collection of functions used in the model.

Cardinality: any number, zero or more

Attribute: activities

Type: ActivityDeclaration

Definition: a unit of work constrained to occur within a certain defined bounds.

Cardinality: any number, zero or more

\begin{tabular}{|l|l|}
\hline Attribute: resources & Type: ResourceDeclaration \\
\hline
\end{tabular}

Definition: a collection of equipment, labor or material being used to perform an activity.

Cardinality: at least one

\begin{tabular}{|l|l|}
\hline Attribute: assertions & Type: Constraint \\
\hline
\end{tabular}

Definition: an expression used to check if condition for the optimization model that need to be valid is respected.

Cardinality: at most one

\begin{tabular}{|l|l|}
\hline Attribute: scripts & Type: Script \\
\hline
\end{tabular}

Definition: a collection of objects of type Script defined and embedded in the optimization model.

Cardinality: any number, zero or more

\begin{tabular}{|l|l|}
\hline Attribute: settings & Type: Setting \\
\hline
\end{tabular}

Definition: a collection of settings defined over properties of the optimization model.

Cardinality: any number, zero or more

\begin{tabular}{|l|l|}
\hline Attribute: declarations & Type: Declaration \\
\hline
\end{tabular}

Definition: an ordered collection of the subtypes of Declaration.

Cardinality: any number, zero or more 


\begin{tabular}{|l|l|}
\hline Attribute: search & Type: SearchProcedure \\
\hline
\end{tabular}

Definition: an optional search procedure defined to find a feasible solution in the scope of the objective.

Cardinality: at most one

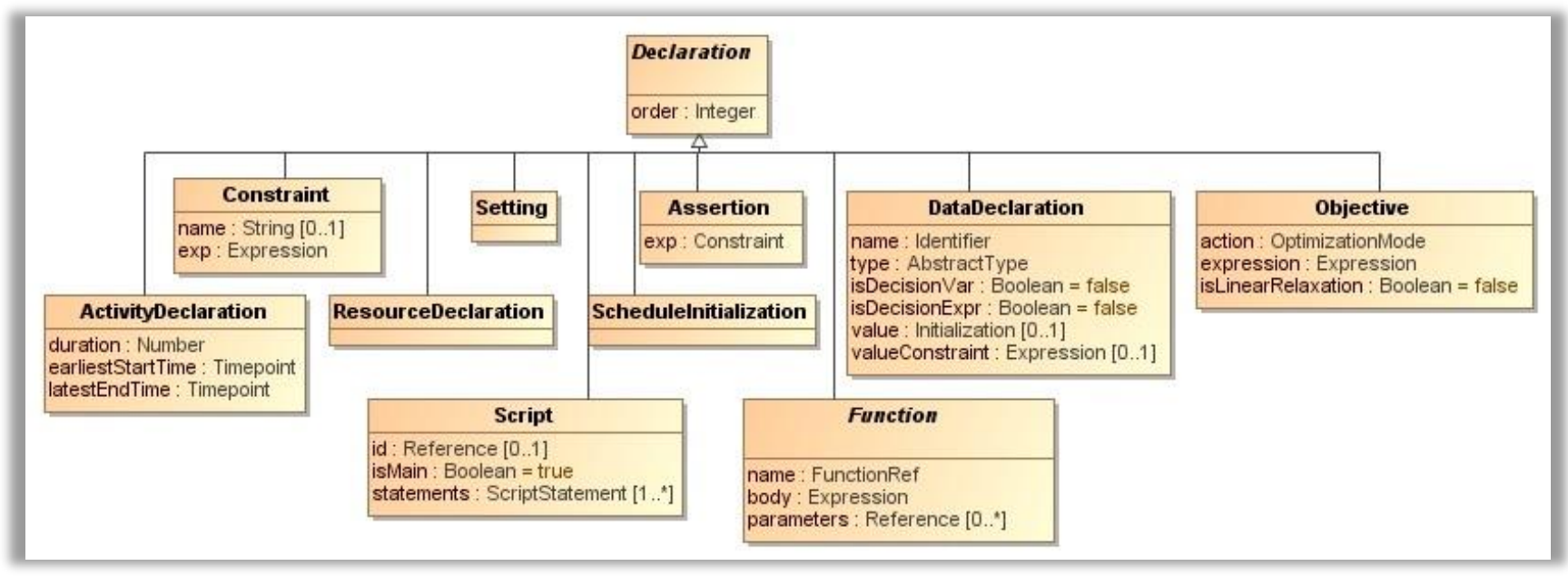

Figure 4: Optimization problem declaration

\subsubsection{Class: Declaration}

Definition: declaration of variables or elements of the model. Declaration is an abstract class defining an ordered collection of subtypes (or subsets declarations) such as DefinedType, DataDeclaration, Constraint, Objective, Function, ActivityDeclaration, ResourceDeclaration, Script, etc.

\section{Properties}

\begin{tabular}{|l|l|}
\hline Attribute: order & Type: Integer \\
\hline
\end{tabular}

Definition: specifies the order in which declarations are made inside an optimization model.

\subsubsection{Class: ActivityDeclaration}

Definition: a unit of work constrained to occur within the bounds defined.

\section{Properties}

\section{Attribute: duration}

Type: Number

Definition: the quantity of time required to perform the activity associated with this declaration.

\section{Attribute: earliestStartTime}

\section{Type: Timepoint}

Definition: the earliest time point at which the activity may start.

\begin{tabular}{|l|l|}
\hline Attribute: latestEndTime & Type: Timepoint \\
\hline
\end{tabular}

Definition: the latest time point at which the activity may end.

\subsubsection{Class: Constraint}

Definition: a Constraint in an optimization model is represented by an expression specifying the limit on the combinations of values for a given objective. 


\section{Properties}

\begin{tabular}{|l|l|}
\hline Attribute: name & Type: String \\
\hline
\end{tabular}

Definition: the name of the Constraint.

Cardinality: at most one

\begin{tabular}{|l|l|}
\hline Attribute: exp & Type: Expression \\
\hline
\end{tabular}

Definition: the expression evaluated in the scope of the Constraint definition.

\subsubsection{Class: Assertion}

Definition: a collection of Boolean expressions that must be evaluated to true; OR an expression used to check if condition for the optimization model that needs to be valid is respected.

Properties

\begin{tabular}{|l|l}
\hline Attribute: exp & Type: Constraint \\
\hline
\end{tabular}

Definition: the expression defining the Assertion that must be checked in the scope of the model verification.

\subsubsection{Class: ResourceDeclaration}

Definition: declaration of a collection of equipment, labor or material being used to perform an activity.

\section{Properties}

none.

\subsubsection{Class: DataDeclaration}

Definition: declaration of a data object.

\section{Properties}

Attribute: name

Type: Identifier

Definition: the name used to refer to the data object.

\begin{tabular}{|l|l|}
\hline Attribute: type & Type: AbstractType \\
\hline
\end{tabular}

Definition: the type of the data object.

\begin{tabular}{|l|l|}
\hline Attribute: isDecisionVar & Type: Boolean \\
\hline
\end{tabular}

Definition: a Boolean indicating whether or not the data object may be modified in the course of execution; AND the value expresses a dimension of the decision space.

\section{Attribute: isDecisionExpr}

Type: Boolean

Definition: a Boolean indicating whether or not the data object is specified by a lexical structure producing its value. 


\begin{tabular}{|l|l|}
\hline Attribute: value & Type: Initialization \\
\hline
\end{tabular}

Definition: the initial value of the data object.

Cardinality: at most one.

\begin{tabular}{|l|l|}
\hline Attribute: valueConstraint & Type: Expression \\
\hline
\end{tabular}

Definition: an optional expression governing the valid values of the data object.

Cardinality: at most one.

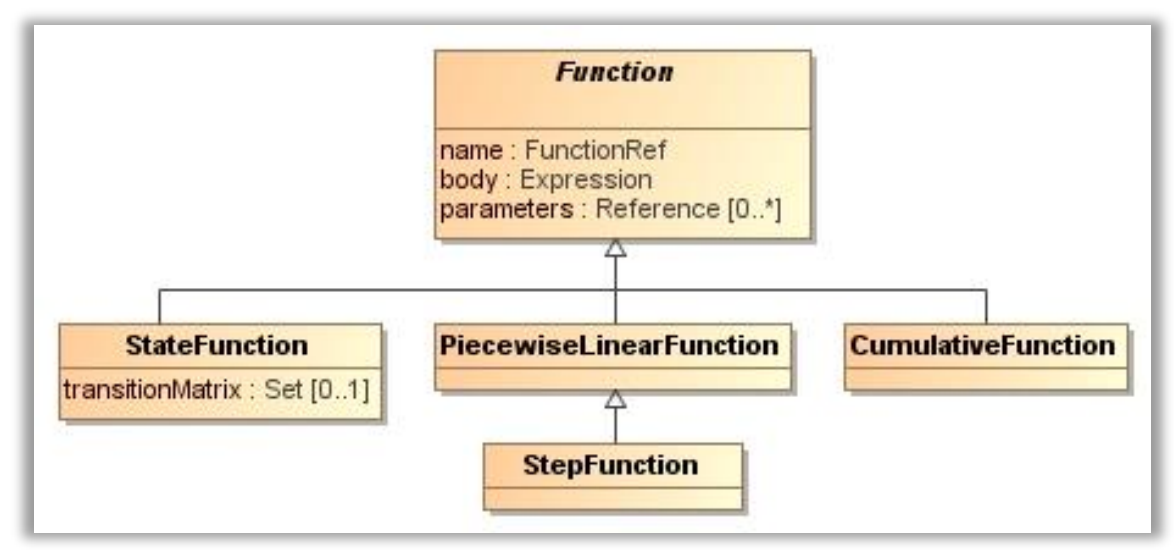

Figure 5: Functions in optimization problems

\subsubsection{Class: Function}

Definition: a function binds a set of parameters (.parameters) in the scope of an expression (.body) and evaluates the Expression producing a value; OR a scoped block of code that can be called with parameters and returns a value.

\section{Properties}

\begin{tabular}{|l|l|}
\hline Attribute: name & Type: Identifier \\
\hline
\end{tabular}

Definition: the name of the function.

\begin{tabular}{|l|l|}
\hline Attribute: body & Type: Expression \\
\hline
\end{tabular}

Definition: the Expression evaluated in the scope of the parameters binding.

\begin{tabular}{|l|l|}
\hline Attribute: parameters & Type: Reference \\
\hline
\end{tabular}

Definition: identifiers bound in the call to the Function.

Cardinality: at least one

\subsubsection{Class: StateFunction}

Definition: a function describing the evolution of a given feature (of the optimization environment) constrained by interval variables of the optimization problem.

\section{Properties}




\begin{tabular}{|l|l|}
\hline Attribute: transitionMatrix & Type: Set \\
\hline
\end{tabular}

Definition: a matrix containing a set of transition time (defined as set of integer numbers) necessary for a resource to switch from a state to another state in a StateFunction.

Cardinality: at most one

\subsubsection{Class: PiecewiseLinearFunction}

Definition: a function that is not purely linear but consist of linear segments with different slopes limited by breakpoints.

\section{Properties}

none.

\subsubsection{Class: StepFunction}

Definition: StepFunction is a special case of PiecewiseLinearFunction where all slopes are equal to 0 and the function domain is integer; AND a step function (or stepwise function) is used, in scheduling, to model the efficiency of a resource over time.

\section{Properties}

none.

\subsubsection{Class: CumulativeFunction}

Definition: a cumulative function expression can be used to model a quantity that varies over time and whose value depends on other decision variables of the problem.

\section{Properties}

none.

\subsubsection{Class: Objective}

Definition: it is the objective function of the optimization problem; AND its value measures the goodness of the solution.

\section{Properties}

\begin{tabular}{|l|l}
\hline Attribute: action & Type: OptimizationMode \\
\hline
\end{tabular}

Definition: the specification of whether the objective function is to be minimized, maximized or solved.

\begin{tabular}{|l|l|}
\hline Attribute: expression & Type: Expression \\
\hline
\end{tabular}

Definition: Expression of the objective function.

\begin{tabular}{|l|l|}
\hline Attribute: isLinearRelaxation & Type: Boolean \\
\hline
\end{tabular}

Definition: a Boolean indicating whether or not the objective is required to be solved by a linear relaxation.

\subsubsection{Class: Setting}

Definition: various options allowing the customization of the behavior of the optimization model.

\section{Properties}


none

\subsubsection{Class: Script}

Definition: a script is a program or function expression that can be embedded in the optimization model and executed from it.

\section{Properties}

\begin{tabular}{|l|l|}
\hline Attribute: id & Type: Reference \\
\hline
\end{tabular}

Definition: the unique (optional) identification of the Script.

Cardinality: at most one

\begin{tabular}{|l|l|}
\hline Attribute: isMain & Type: Boolean \\
\hline
\end{tabular}

Definition: a Boolean indicating whether or not the Script is defined as the main method of an optimization model.

\begin{tabular}{|l|l|}
\hline Attribute: statements & Type: ScriptStatement \\
\hline
\end{tabular}

Definition: the statement describing the content of the Script.

Cardinality: at least one

\subsubsection{Class: SearchProcedure}

Definition: an optional search procedure defined to find feasible solution in the scope of the objective.

\section{Properties}

none.

\subsection{Data type concepts}

The OptimizationDataTypes concepts package identifies and specifies different types of data existing in an optimization problem model. The concepts are depicted in Figure 6, Figure 7, Figure 8 and Figure 9.

\subsubsection{Class: AbstractType}

Definition: a type descriptor for any type from which sub-classes may be derived, but which itself cannot be instantiated directly; AND instances of AbstractType are type descriptors.

\section{Properties}

none. 


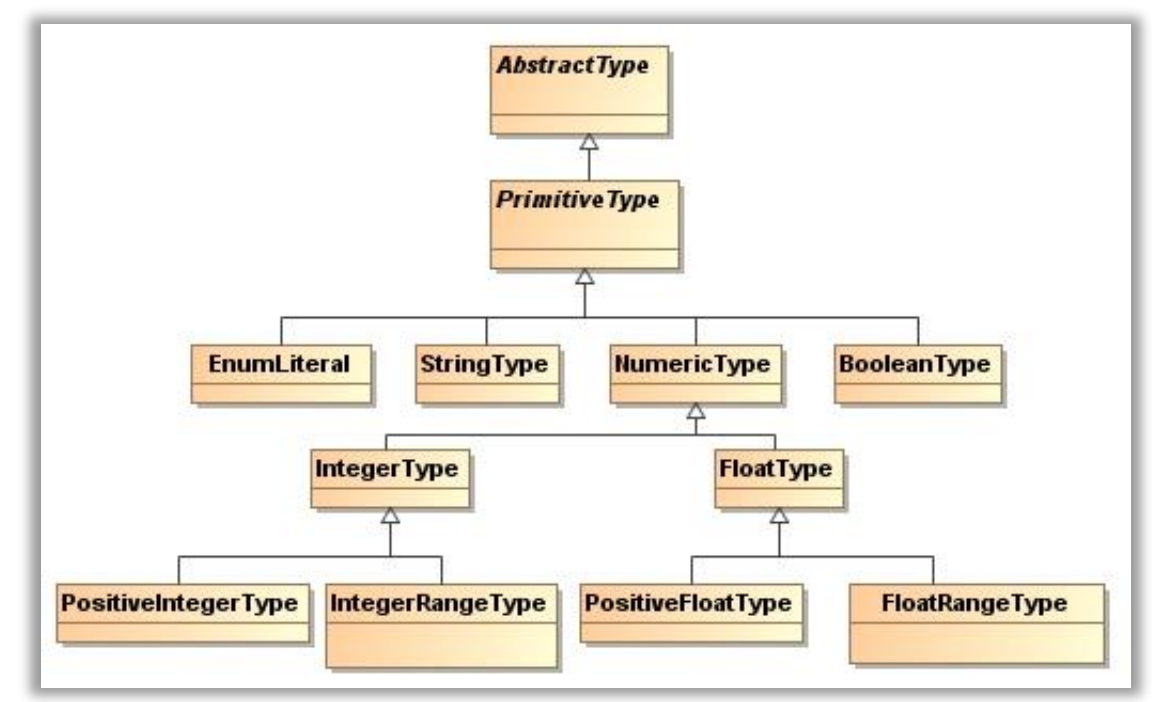

Figure 6: Optimization AbstractPrimitive data types

\subsubsection{Class: PrimitiveType}

Definition: a type descriptor for any type where the value provides identity.

\section{Properties}

none.

\subsubsection{Class: EnumLiteral}

Definition: an element of an EnumerationType.

\section{Properties}

none.

\subsubsection{Class: BooleanType}

Definition: a type whose values are True and False.

\section{Properties}

none.

\subsubsection{Class: StringType}

Definition: a data object type that represents sequences of characters.

\section{Properties}

none.

\subsubsection{Class: NumericType}

Definition: a type used to handle numbers in various representations.

\section{Properties}

none. 


\subsubsection{Class: IntegerType}

Definition: an integer numeric type defined by a positive or negative counting number (that can be written without a fractional component) or zero.

\section{Properties}

none.

\subsubsection{Class: PositiveIntegerType}

Definition: a positive integer type.

\section{Properties}

none.

\subsubsection{Class: IntegerRangeType}

Definition: an ordered set of integer types limited by a lower and an upper bounds, and whose values can be stored inside a variable of type IntegerType.

\section{Properties}

none.

\subsubsection{Class: FloatType}

Definition: a float numeric type defined by a positive or negative number written with a fractional component (value); OR a number having digits on both sides of a decimal point.

\section{Properties}

none.

\subsubsection{Class: PositiveFloatType}

Definition: a positive numeric type written with fractional parts.

\section{Properties}

none.

\subsubsection{Class: FloatRangeType}

Definition: an ordered set of float types limited by a lower and an upper bounds.

\section{Properties}

None.

\subsubsection{Class: DefinedType}

Definition: an AbstractType defined in the scope of the model.

\section{Properties}

none. 


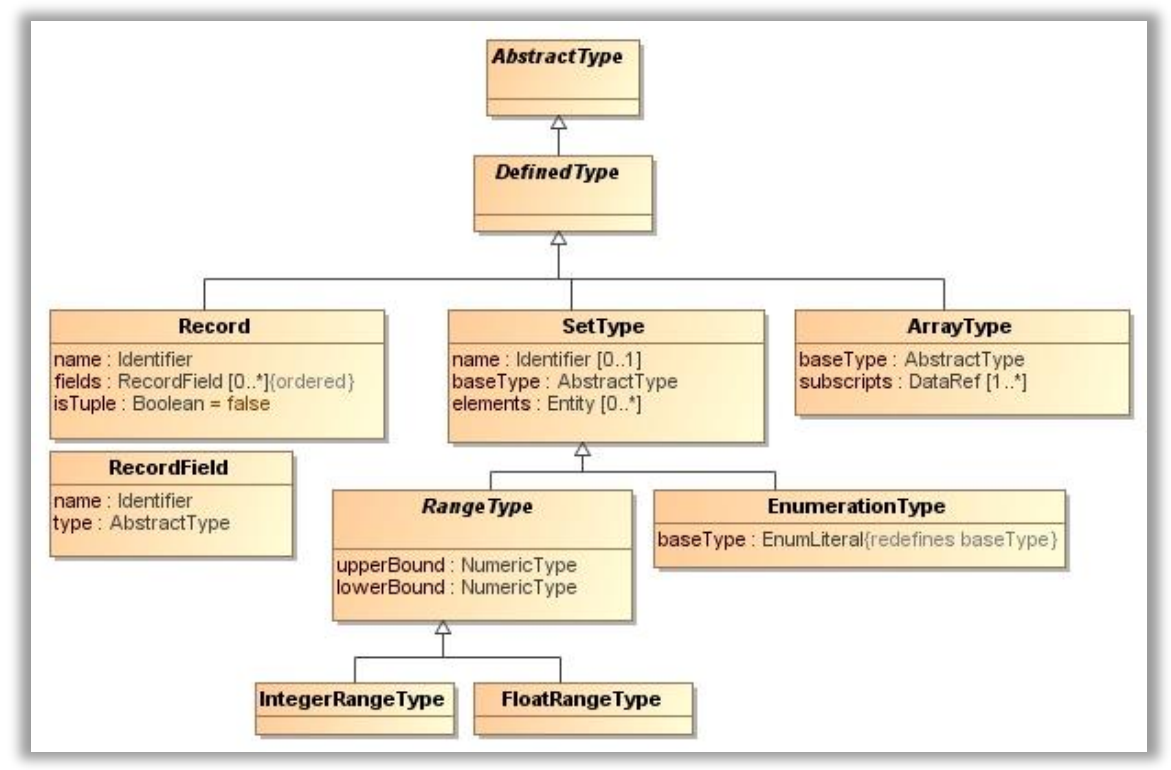

Figure 7: Optimization AbstractDefined data types

\subsubsection{Class: Record}

Definition: a DefinedType that defines a record structure, that is, named and ordered Record fields.

\section{Properties}

\begin{tabular}{|l|l|}
\hline Attribute: name & Type: Identifier \\
\hline
\end{tabular}

Definition: a name identifying the record structure.

\begin{tabular}{|l|l|}
\hline Attribute: fields & Type: RecordField \\
\hline
\end{tabular}

Definition: an ordered collection of the RecordFields of the record.

Cardinality: any number, zero or more

\begin{tabular}{|l|l|}
\hline Attribute: isTuple & Type: Boolean \\
\hline
\end{tabular}

Definition: a data structure containing a given number of elements in a given order.

\subsubsection{Class: RecordField}

Definition: a field allocated to the record that being referred.

\section{Properties}

\begin{tabular}{|l|l|}
\hline Attribute: name & Type: String \\
\hline
\end{tabular}

Definition: the name of the RecordField.

\begin{tabular}{|l|l|}
\hline Attribute: type & Type: AbstractType \\
\hline
\end{tabular}

Definition: the type of the RecordField.

\subsubsection{Class: SetType}

Definition: a DefinedType that describes a set by enumerating its elements. 


\section{Properties}

\begin{tabular}{|l|l|}
\hline Attribute: name & Type: String \\
\hline
\end{tabular}

Definition: an optional name for the set.

Cardinality: at most one

Attribute: baseType

Type: AbstractType

Definition: the type of the elements of the set.

\begin{tabular}{|l|l|}
\hline Attribute: elements & Type: Entity \\
\hline
\end{tabular}

Definition: the elements of the set.

Cardinality: any number, zero or more

\subsubsection{Class: RangeType}

Definition: a SetType whose BaseType is NumericType.

\section{Properties}

\section{Attribute: upperBound}

Type: NumericType

Definition: the largest element of the set.

\begin{tabular}{|l|l|}
\hline Attribute: lowerBound & Type: NumericType
\end{tabular}

Definition: the smallest element of the set.

\subsubsection{Class: IntegerRangeType}

Definition: a RangeType whose BaseType is IntegerType

Properties

none

\subsubsection{Class: FloatRangeType}

Definition: a RangeType whose BaseType is FloatType.

\section{Properties}

none

\subsubsection{Class: EnumerationType}

Definition: a SetType whose BaseType is EnumLiteral

\section{Properties}

\section{Attribute: baseType}

Type: EnumLiteral

Definition: the type of the elements of the EnumerationType.

\subsubsection{Class: ArrayType}

Definition: a DefinedType constructed from an indexed collection of its .baseType. 


\section{Properties}

\begin{tabular}{|l|l|}
\hline Attribute: baseType & Type: AbstractType
\end{tabular}

Definition: the type of the elements of the array.

\begin{tabular}{|l|l|}
\hline Attribute: subscripts & Type: DataRef \\
\hline
\end{tabular}

Definition: similar to the array index, subscripts can be defined as the inferior (symbol or number) used to identify elements in an array.

Cardinality: at least one

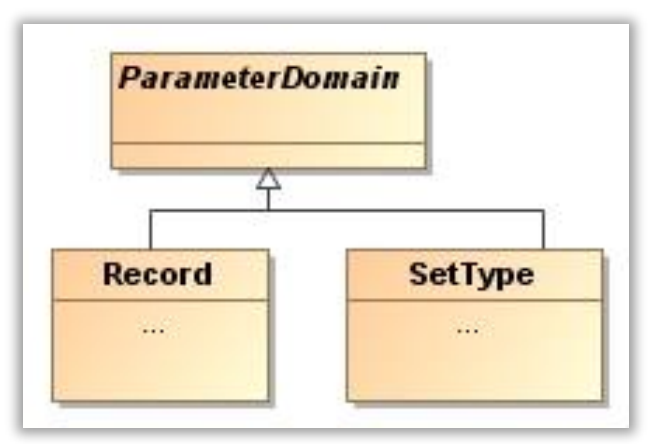

Figure 8: Optimization Parameters domain

\subsubsection{Class: ParameterDomain}

Definition: the set of possible values that can be assigned to an element (or variable) being referred in a Record, in the scope of an objective.

\section{Properties}

none.

\subsubsection{Class: Record}

Definition: a DefinedType that defines a record structure, that is, named and ordered Record fields (See section 3.2.1.2.1).

\subsubsection{Class: SetType}

Definition: a DefinedType describing a set by enumerating its elements (See section 3.2.1.2.2)

\begin{tabular}{|l|}
\hline \multicolumn{1}{|c|}{ Interval } \\
\hline isOptional : Boolean = false \\
bounds: RangeType \\
size: NumericType \\
intensity: StepFunction \\
\hline
\end{tabular}

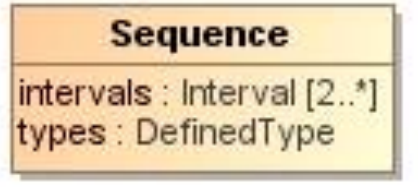

Figure 9: Interval in optimization data types

\subsubsection{Class: Sequence}


Definition: describes a set of data objects lying within an interval.

Properties

Attribute: intervals

Type: Interval

Definition: the interval of the sequence being referred.

Cardinality: at least two

\begin{tabular}{|l|l|}
\hline Attribute: types & Type: DefinedType
\end{tabular}

Definition: the types of the data object in the scope of the sequence being referred.

\subsubsection{Class: Interval}

Definition: represents an interval of time during which something happens, and whose position in time is an unknown to the scheduling problem.

Properties

\begin{tabular}{|l|l|}
\hline Attribute: isOptional & Type: Boolean
\end{tabular}

Definition: a Boolean indicating whether or not the interval is mandatory in the solution definition.

\begin{tabular}{|l|l|}
\hline Attribute: bounds & Type: RangeType \\
\hline
\end{tabular}

Definition: the bounds of the interval defined by a start time and an end time within a certain range.

\begin{tabular}{|l|l|}
\hline Attribute: size & Type: Number \\
\hline
\end{tabular}

Definition: the size of the interval.

\begin{tabular}{|l|l|}
\hline Attribute: intensity & Type: StepFunction \\
\hline
\end{tabular}

Definition: the intensity of the interval variables defined by a stepwise linear function.

\subsection{Expression concepts}

The OptimizationExpressions package provides the context for identifying different types of expressions used in an optimization problem model. The concepts are depicted in Figure 10, Figure11, Figure 12, Figure13, Figure14 and Figure15.

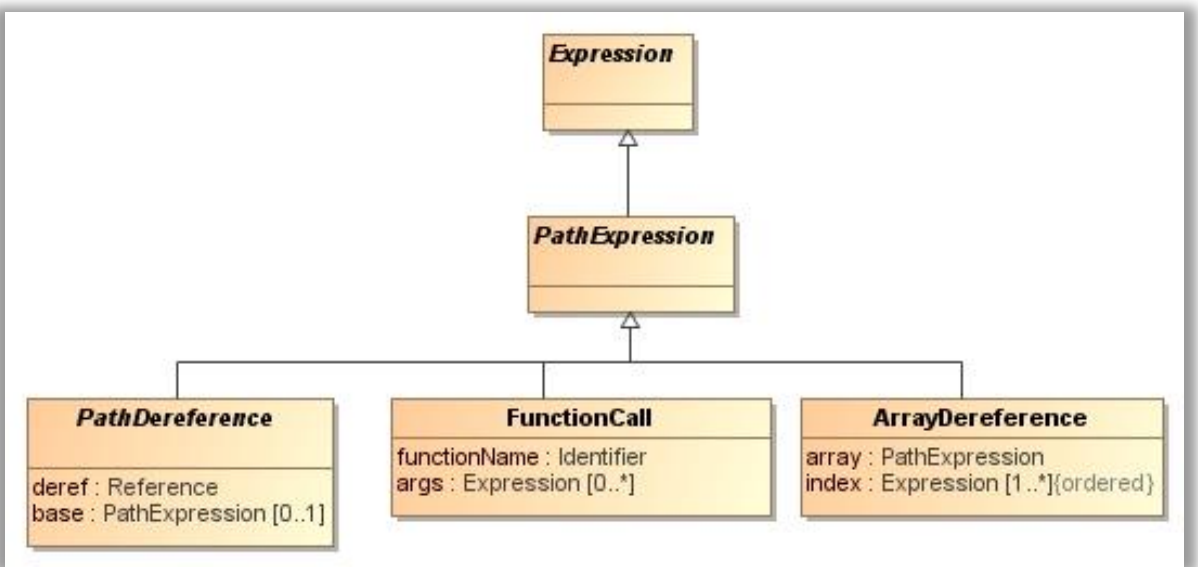


Figure 10: PathExpression in optimization problem

\subsubsection{Class: Expression}

Definition: an abstract class representing a lexical structure that may be evaluated to produce a value conforming to AbstractType.

\section{Properties}

none.

\subsubsection{Class: PathExpression}

Definition: an abstract Expression defining the path to a value.

\section{Properties}

none

\subsubsection{Class: PathDereference}

Definition: a PathExpression (may be partial) that navigates to a Reference.

\section{Properties}

\begin{tabular}{|l|l|}
\hline Attribute: deref & Type: Reference \\
\hline
\end{tabular}

Definition: the partial PathExpression navigating to a Reference specified by PathExpression.deref.

\begin{tabular}{|l|l|}
\hline Attribute: base & Type: PathExpression \\
\hline
\end{tabular}

Definition: the partial PathExpression navigating to the base of the PathExpression.

Cardinality: at most one

\subsubsection{Class: FunctionCall}

Definition: a PathExpression that takes some arguments as input and whose execution (call) returns a result.

Properties

\begin{tabular}{|l|l|}
\hline Attribute: functionName & Type: FunctionRef \\
\hline
\end{tabular}

Definition: the name of the Function being called.

\begin{tabular}{|l|l|}
\hline Attribute: args & Type: Expression \\
\hline
\end{tabular}

Definition: the expression of the arguments being input to the Function.

Cardinality: any number, zero or more

\subsubsection{Class: ArrayDereference}

Definition: a reference into an array.

Properties

\begin{tabular}{|l|l}
\hline Attribute: array & Type: PathExpression \\
\hline
\end{tabular}

Definition: the partial PathExpression navigating to an array. 


\begin{tabular}{|l|l|}
\hline Attribute: index & Type: Expression \\
\hline
\end{tabular}

Definition: the offset into the array referenced by ArrayDereference.array.

Cardinality: at least one

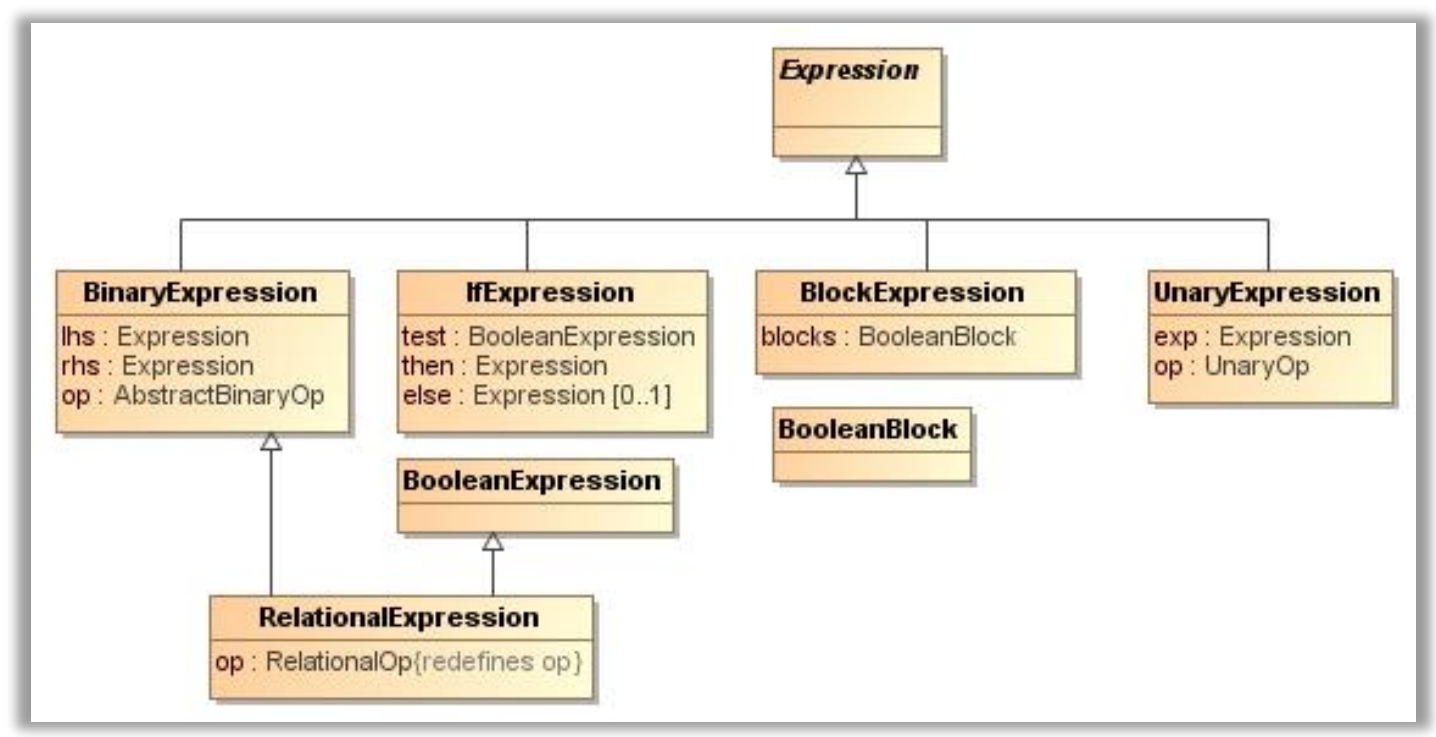

Figure 11: Expressions in optimization problem

\subsubsection{Class: BinaryExpression}

Definition: an expression formed from the application of a BinaryOp to two subexpressions.

\section{Properties}

\begin{tabular}{|l|l|}
\hline Attribute: lhs & Type: Expression \\
\hline
\end{tabular}

Definition: an Expression on the left-hand side of a binary operator specified by BinaryExpression.op.

\begin{tabular}{|l|l|}
\hline Attribute: rhs & Type: Expression \\
\hline
\end{tabular}

Definition: an Expression on the right-hand side of a binary operator specified by BinaryExpression.op.

\begin{tabular}{|l|l|}
\hline Attribute: op & Type: AbstractBinaryOp \\
\hline
\end{tabular}

Definition: the operator of the BinaryExpression, of type AbstractBinaryOp.

\subsubsection{Class: RelationalExpression}

Definition: an expression built using operators of type RelationalOp and returning a value of type BooleanType.

\section{Properties}

\begin{tabular}{|l|l|}
\hline Attribute: op & Type: RelationalOp \\
\hline
\end{tabular}

Definition: the operator of the RelationalExpression of type RelationalOp.

\subsubsection{Class: BlockExpression}

Definition: an expression formed from a set of block. 


\section{Properties}

\begin{tabular}{|l|l|}
\hline Attribute: blocks & Type: BooleanBlock \\
\hline
\end{tabular}

Definition: the blocks describing the condition inside the BlockExpression.

\subsubsection{Class: BooleanBlock}

Definition: the block containing a condition which execution returns a Boolean.

Properties

none

\subsubsection{Class: IfExpression}

Definition: an Expression describing if/then /else statement.

\section{Properties}

\begin{tabular}{|l|l|}
\hline Attribute: test & Type: BooleanExpression \\
\hline
\end{tabular}

Definition: the condition deciding whether the 'then' or 'else' branch is to be evaluated.

\begin{tabular}{|l|l|}
\hline Attribute: then & Type: Expression \\
\hline
\end{tabular}

Definition: an expression to be evaluated when IfExpression.condition evaluates to true.

\begin{tabular}{|l|l|}
\hline Attribute: else & Type: Expression \\
\hline
\end{tabular}

Definition: an optional expression to be evaluated when IfExpression.condition evaluates to false.

Cardinality: at most one

\subsubsection{Class: UnaryExpression}

Definition: an expression formed as the application of a unary operator to an expression.

\section{Properties}

\begin{tabular}{|l|l|}
\hline Attribute: exp & Type: Expression \\
\hline
\end{tabular}

Definition: the subexpression to which the unary operation, UnaryExpression.op, is applied.

\begin{tabular}{|l|l|}
\hline Attribute: op & Type: UnaryOp \\
\hline
\end{tabular}

Definition: the operator of the UnaryExpression, of type UnaryOp. 


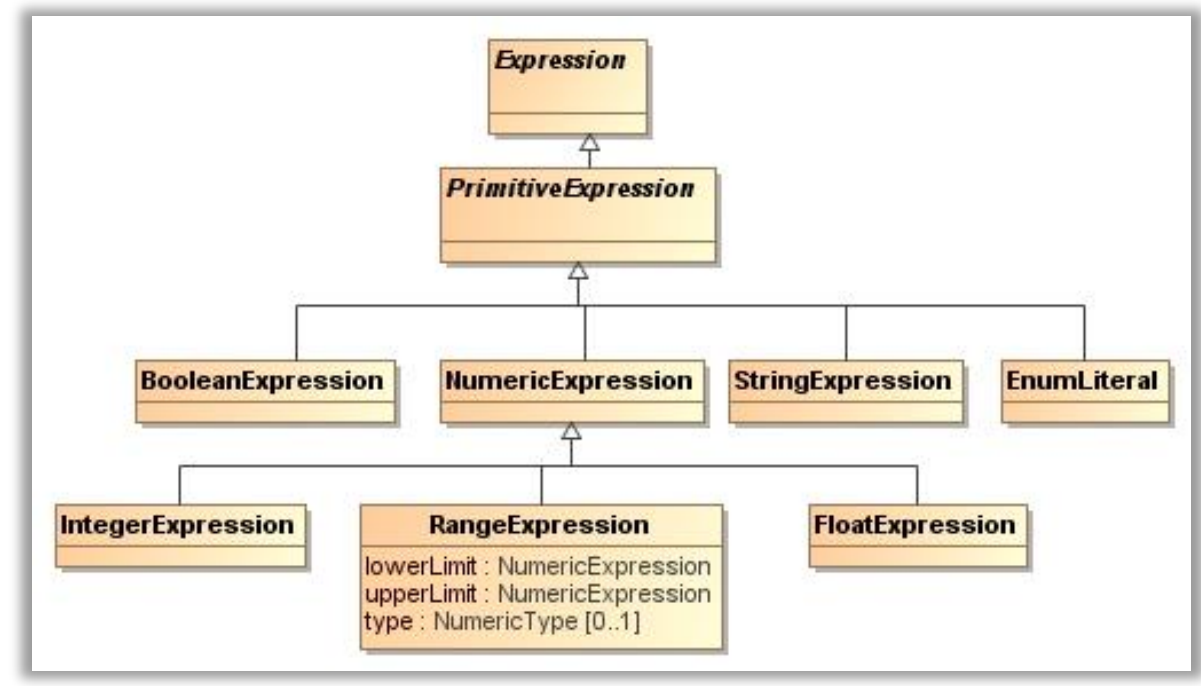

Figure 12: Primitive expressions in optimization problem

\subsubsection{Class: PrimitiveExpression}

Definition: an expression that evaluates to a primitive.

\section{Properties}

none.

\subsubsection{Class: BooleanExpression}

Definition: a PrimitiveExpression that evaluates to a Boolean.

\section{Properties}

none.

\subsubsection{Class: NumericExpression}

Definition: a PrimitiveExpression that evaluates to a Numeric.

\section{Properties}

none.

\subsubsection{Class: IntegerExpression}

Definition: a NumericExpression that evaluates to an Integer.

\section{Properties}

none.

\subsubsection{Class: RangeExpression}

Definition: an AllRange defining the lower and upper bounds on the Range.

\section{Properties}




\begin{tabular}{|l|l|}
\hline Attribute: lowerLimit & Type: NumericExpression \\
\hline
\end{tabular}

Definition: the smallest element of the Range.

\begin{tabular}{|l|l|}
\hline Attribute: upperLimit & Type: NumericExpression \\
\hline
\end{tabular}

Definition: the largest element of the Range.

\begin{tabular}{|l|l|}
\hline Attribute: type & Type: NumericType
\end{tabular}

Definition: the (optional) type of the element defined within the Range.

Cardinality: at least one

\subsubsection{Class: FloatExpression}

Definition: a PrimitiveExpression that evaluates to a Float.

\section{Properties}

none.

\subsubsection{Class: StringExpression}

Definition: a PrimitiveExpression that evaluates to a String.

\section{Properties}

none.

\subsubsection{Class: EnumLiteral}

Definition: similar to the one defined in section 3.2.1.1.1 under PrimitiveAbstractType.

\section{Properties}

none.

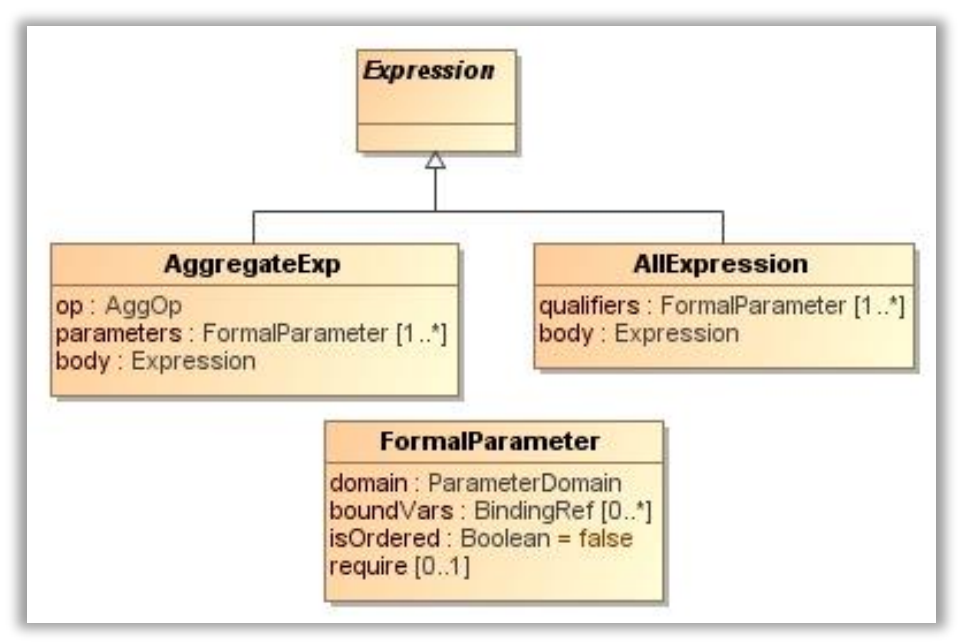

Figure 13: AllExpression and AggregateExpression

\subsubsection{Class: AllExpression}


Definition: an expression formed as the application of the keyword "all" that allows the usage of only part of an array with functions that take array parameters.

\section{Properties}

\begin{tabular}{|l|l|}
\hline Attribute: qualifiers & Type: FormalParameter \\
\hline
\end{tabular}

Definition: the formal parameter that is being referred in the expression.

Cardinality: at least one

\begin{tabular}{|l|l|}
\hline Attribute: body & Type: Expression \\
\hline
\end{tabular}

Definition: the body of the Expression.

\subsubsection{Class: AggregateExpression}

Definition: an Expression formed from the application of an AggOp to two subexpressions.

\section{Properties}

\begin{tabular}{|l|l|}
\hline Attribute: op & Type: AggOp \\
\hline
\end{tabular}

Definition: the operator of the AggregateExpression, of type AggOp.

\begin{tabular}{|l|l|}
\hline Attribute: parameters & Type: FormalParameters \\
\hline
\end{tabular}

Definition: the parameters evaluated inside the AggregateExpression.

Cardinality: at least one

\begin{tabular}{|l|l|}
\hline Attribute: body & Type: Expression \\
\hline
\end{tabular}

Definition: the body of the AggregateExpression.

\subsubsection{Class: FormalParameter}

Definition: An abstract representation (usually composed of a name and a type) of a parameter expected to be called in the scope of an expression.

\section{Properties}

\section{Attribute: domain}

\section{Type: ParameterDomain}

Definition: the set of possible values over which the FormalParameter may range.

\begin{tabular}{|l|l|}
\hline Attribute: boundVars & Type: BindingRef \\
\hline
\end{tabular}

Definition: a list of BindingRef (variables) used in the body of an AggregateExpression or AllExpression.

Cardinality: at least one

\begin{tabular}{|l|l|}
\hline Attribute: isOrdered & Type: Boolean \\
\hline
\end{tabular}

Definition: a Boolean indicating whether or not the collection of values in the range of the FormalParameter is ordered or not.

\begin{tabular}{|l|l|}
\hline Attribute: require & Type: Expression \\
\hline
\end{tabular}

Definition: a constraint governing valid values of the .boundVars. 


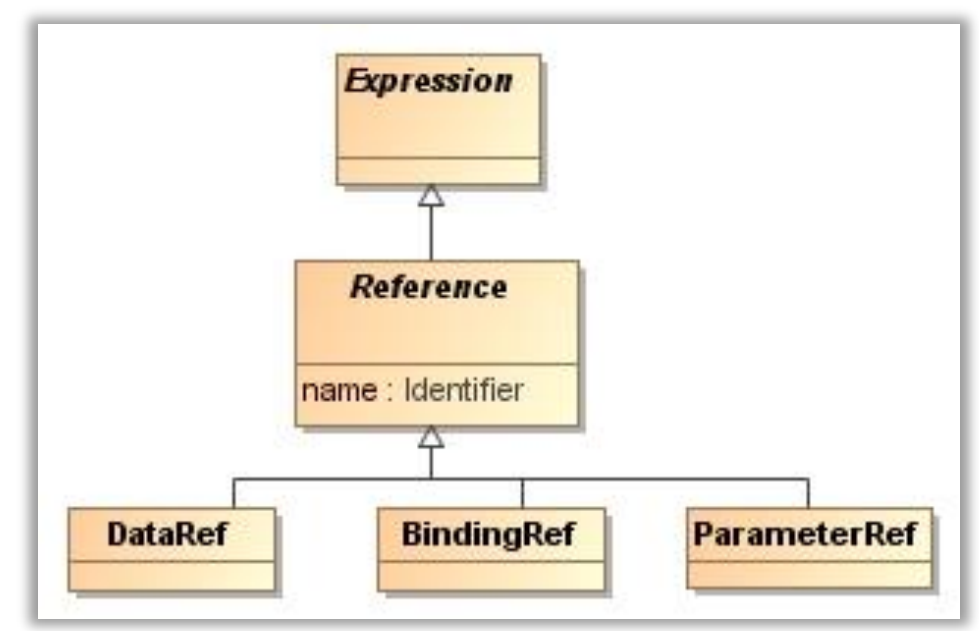

Figure 14: References in optimization problems

\subsubsection{Class: Reference}

Definition: a relational that takes a Binary and returns a Boolean.

\section{Properties}

\begin{tabular}{|l|l|}
\hline Attribute: name & Type: Identifier \\
\hline
\end{tabular}

Definition: the name that identifies the reference.

\subsubsection{Class: DataRef}

Definition: a reference to a DataObject.

\section{Properties}

none.

\subsubsection{Class: BindingRef}

Definition: a reference to a VariableBinding.

\section{Properties}

none.

\subsubsection{Class: ParameterRef}

Definition: a reference introduced in a FormalParameter.

\section{Properties}

none.

\subsubsection{Class: VariableBinding}

Definition: specifies the binding of a variable to a declared DataObject.

\section{Properties}




\begin{tabular}{|l|l|}
\hline Attribute: vars & Type: BindlingRef \\
\hline
\end{tabular}

Definition: the reference binding a variable to its DataObject.

Cardinality: at least one

\begin{tabular}{|l|l|}
\hline Attribute: domain & Type: Expression \\
\hline
\end{tabular}

Definition: an expression that specifies the domain of the variables.

\subsubsection{Class: DataObject}

Definition: a data at meta-level below the OPL metamodel; AND it is an instance of an AbstractType whose value must conform to the type declared in the DataDeclaration.

\section{Properties}

none.

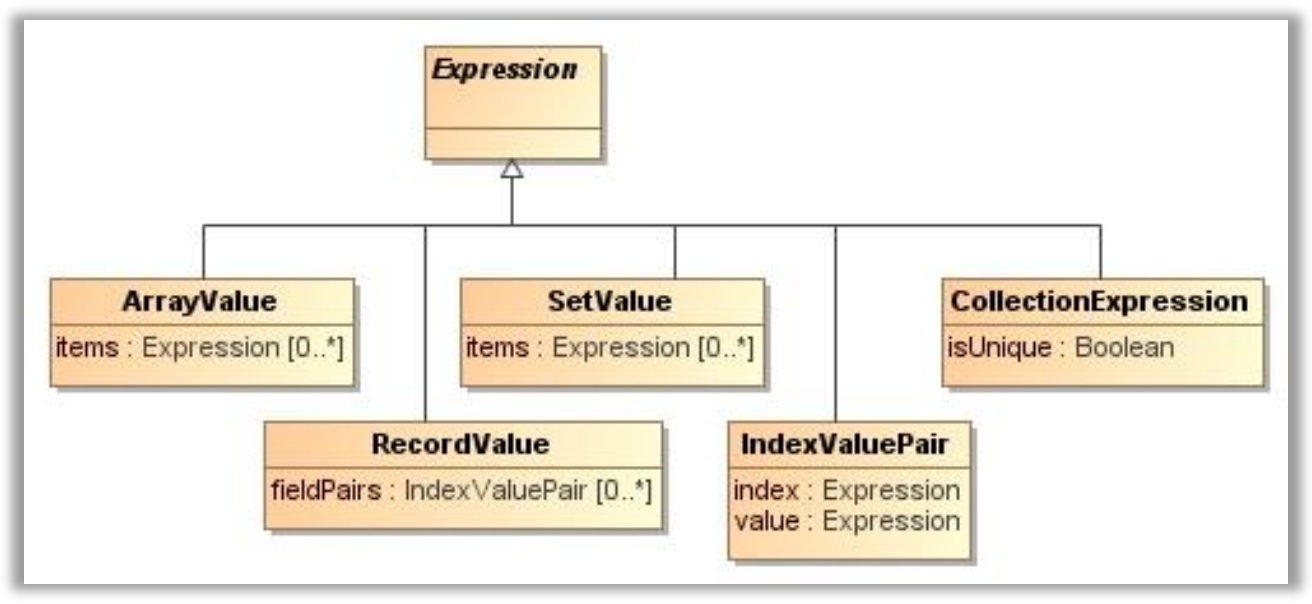

Figure 15: Expressions in optimization problems

\subsubsection{Class: ArrayValue}

Definition: an Expression that evaluates to an ArrayType indexed by offset into the ordered collection.

\section{Properties}

\begin{tabular}{|l|l|}
\hline Attribute: items & Type: Expression \\
\hline
\end{tabular}

Definition: an ordered collection of Expression, each of which evaluates to an element of the Array; AND the resulting ArrayType object is indexed by offset into the ordered collection (i.e. $0,1,2 \ldots$ ).

Cardinality: any number, zero or more

\subsubsection{Class: RecordValue}

Definition: an Expression that evaluates to a RecordType instance.

\section{Properties}

\begin{tabular}{|l|l|}
\hline Attribute: fieldPairs & Type: IndexValuePair \\
\hline
\end{tabular}

Definition: a collection of pairs consisting of a record field name and a value. 
Cardinality: any number, zero or more

\subsubsection{Class: SetValue}

Definition: an Expression that evaluates to a SetType instance.

\section{Properties}

Attribute: items

Type: Expression

Definition: the elements of the SetType.

Cardinality: any number, zero or more

\subsubsection{Class: IndexValuePair}

Definition: a pair of values consisting of a field name and value.

\section{Properties}

\begin{tabular}{|l|l|}
\hline Attribute: index & Type: Expression \\
\hline
\end{tabular}

Definition: an Expression evaluating to the name of the field.

\begin{tabular}{|l|l|}
\hline Attribute: value & Type: Expression \\
\hline
\end{tabular}

Definition: an Expression evaluating to the value of the field.

\subsubsection{Class: CollectionExpression}

Definition: an Expression formed from a collection of DataObject

\section{Properties}

\begin{tabular}{|l|l|}
\hline Attribute: isUnique & Type: Boolean \\
\hline
\end{tabular}

Definition: a Boolean indicating whether or not the collection is unique.

\subsection{Operator concepts}

This section of the metamodel related to the OptimizationOperators package introduces the common concepts surrounding operator's usage in optimization problem definition. These concepts are depicted in Figure 16. 


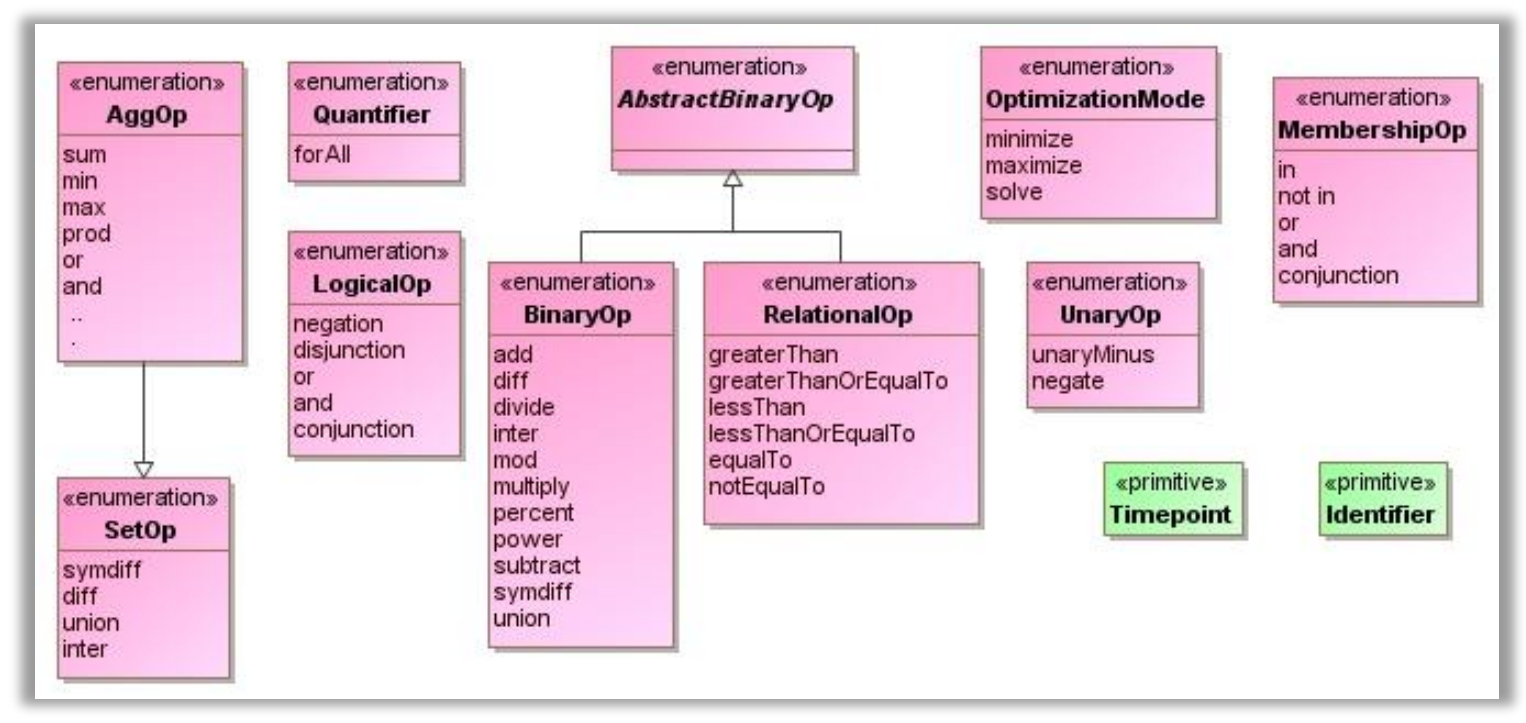

Figure 16: Operators in optimization problems

Concepts in the OptimizationOperators' package appear as Enumeration classes. In an enumeration class box, the lower partition is the list of the Named Individuals that are members. In Figure 14, the enumeration classes are: AggOp, SetOp, LogicalOp, AbstractBinaryOp, BinaryOp, RelationalOp, UnaryOp, MembershipOp, OptimizationMode, and Quantifier. In addition, Timepoint and Identifier are defined as primitive operators.

\subsubsection{Enumeration Class: AggOp}

Definition: specifies the set of operators used to construct expressions of type AggregateExpression

\section{Named Individual Members}

\section{Member: sum}

Definition: the operator used to aggregate, by the mathematical process of addition, two or more numbers, magnitudes, quantities, or expressions.

\section{Member: $\min$}

Definition: the operator used to find the value of an Expression where a minimum occurs.

\section{Member: $\max$}

Definition: the operator used to find the value of an Expression where a maximum occurs

\section{Member: prod}

Definition: the operator used to construct product of two or more expressions together.

\section{Member: or}

Definition: the operator used to connect statements or expressions representing alternative constraints; OR a Boolean operator that returns a positive result when either or both operands are positive.

\section{Member: and}


Definition: the operator used to connect statements or expressions representing cumulative constraints; OR a Boolean operator that returns a positive result when both operands are positive.

\subsubsection{Enumeration Class: SetOp}

Definition: specifies the set of operators used to construct collection or group of similar optimization data designed to be used together

\section{Named Individual Members}

\section{Member: union}

Definition: The operator used to construct a collection (or a set) whose members are elements from two or more other sets.

\section{Member: inter}

Definition: the operator used to compute a set that contains only elements share by two or more other sets.

\section{Member: symdiff}

Definition: the operator used to construct a set containing elements of the difference between the union and the intersection (inter) of two other sets.

\section{Member: diff}

Definition: the operator used to subtract elements of a given set from another one.

\subsubsection{Enumeration Class: LogicalOp}

Definition: specifies the set of operators used to construct logical statement in an optimization problem.

\section{Named Individual Members}

\section{Member: negation}

Definition: the operator used to make a negative statement, or to specify a logical operation of negating.

\section{Member: inclusive disjunction}

Definition: the operator used to construct a compound statement, which is true if and only if at least a number of alternatives is true.

\section{Member: exclusive disjunction}

Definition: the operator used to construct a compound statement, which is true if and only if one and only one of a number of alternatives is true.

\section{Member: or}

Definition: similar to the one defined in section 3.4.1 on AggOp.

\section{Member: and}

Definition: similar to the one defined in section 3.4.1 on AggOp.

\section{Member: conjunction}


Definition: the operator used to construct a compound statement, which is true if and only if all of its component statements are true.

\subsubsection{Enumeration Class: AbstractBinaryOp}

AbstractBinaryOp generalizes two types of operators identified as: BinaryOp and RelationalOp.

\subsubsection{Enumeration Class: BinaryOp}

Definition: specifies the set of operators used to construct statements or expressions relating two logical or mathematical elements; OR the set of operators used to construct expressions of type BinaryExpression.

\section{Named Individual Members}

\section{Member: add}

Definition: the operator used to join or combine (numbers, quantities...) by the arithmetic operation of addition; OR the operator used to find a sum in arithmetic.

\section{Member: diff}

Definition: similar to the one defined in section 3.4.1.1 on SetOp.

\section{Member: divide}

Definition: the operator used to separate an element into equal parts by the process of mathematical integer division.

\section{Member: inter}

Definition: similar to the one defined in section 3.4.1.1 on SetOp.

\section{Member: $\bmod$}

Definition: the operator that evaluates the remainder of a mathematical integer division in an optimization problem.

\section{Member: multiply}

Definition: the operator used to find a product of, by the process of multiplication.

\section{Member: percent}

Definition: the operator used to find a proportion in relation to a whole (usually defined as the amount per hundred).

\section{Member: power}

Definition: the operator used to indicate the number of times a quantity is multiplied by itself.

\section{Member: subtract}

Definition: the operator used to calculate the difference between two numbers or quantities by the arithmetic operation of subtraction.

\section{Member: symdiff}

Definition: similar to the one defined in section 3.4.1.1 on SetOp. 


\section{Member: union}

Definition: similar to the one defined in section 3.4.1.1 on SetOp.

\subsubsection{Enumeration Class: RelationalOp}

Definition: this enumeration contains the set of operators used to indicate or specify some relations in the scope of mathematical expressions.

\section{Named Individual Members}

\section{Member: greaterThan}

Definition: an algebraic relational operator showing inequality between two subexpressions; AND a statement indicating that the value of a subexpression (or quantity) preceding the operator is greater than the value of the subexpression (or quantity) following the operator.

\section{Member: greaterThanOrEqualTo}

Definition: an algebraic relational operator showing inequality between two subexpressions; AND a statement indicating that the value of a subexpression (or quantity) preceding the operator is greater than or equal to the value of the subexpression (or quantity) following the operator.

\section{Member: lessThan}

Definition: an algebraic relational operator showing inequality between two subexpressions; AND a statement indicating that the value of a subexpression (or quantity) preceding the operator is less than the value of the subexpression (or quantity) following the operator.

\section{Member: less ThanOrEqualTo}

Definition: an algebraic relational operator showing inequality between two subexpressions; AND a statement indicating that the value of a subexpression (or quantity) preceding the operator is less than or equal to the value of the subexpression (or quantity) following the operator.

\section{Member: equalTo}

Definition: an algebraic relational operator showing equality between two subexpressions; AND a statement indicating that the value of a subexpression (or quantity) preceding the operator is equal to (or as great as) the value of the subexpression (or quantity) following the operator.

\section{Member: notEqualTo}

Definition: an algebraic relational operator showing inequality between two subexpressions; AND a statement indicating that the value of a subexpression (or quantity) preceding the operator is not equal to the value of the subexpression (or quantity) following the operator.

\subsubsection{Enumeration Class: UnaryOp}

Definition: specifies enumeration of operators used to construct statements or expressions consisting of or involving a single element or component; OR the type of operator used in mathematical operations where only one element is used to yield a single result.

\section{Named Individual Members}




\section{Member: unaryMinus}

Definition: specifies a unary operator which negates the arguments it applies to.

\section{Member: negate}

Definition: specifies the operator used to define negation, which is an operation on the value of a proposition that produces a value of true when its operand is false and a value of false when its operand is true.

\subsubsection{Enumeration Class: MembershipOp}

Definition: the list of operators used to define the state of an optimization data object being member of a set or a given range.

\section{Named Individual Members}

Member: in

Definition: the operator used to define the membership of an element to a set or a range within a certain limits, bounds or area.

\section{Member: not in}

Definition: the operator used to define the non-membership of an element to a set or a range within a certain limits, bounds or area.

\section{Member: or}

Definition: similar to the one defined in section 3.4.2 on LogicalOp.

\section{Member: and}

Definition: similar to the one defined in section 3.4.2 on LogicalOp.

\section{Member: conjunction}

Definition: similar to the one defined in section 3.4.2 on LogicalOp.

\subsubsection{Enumeration Class: OptimizationMode}

Definition: enumeration of the different modes by which an objective function is to be optimized or computed.

\section{Named Individual Members}

\section{Member: minimize}

Definition: the optimization mode used to find the smallest value of an objective function either within a given range or on the entire domain of the function.

\section{Member: maximize}

Definition: the optimization mode used to find the largest value of an objective function either within a given range or on the entire domain of the function.

\section{Member: solve}


Definition: the optimization mode used to find what values fulfill the condition stated in an objective function that is subject to a given (set of) constraint(s).

\subsubsection{Enumeration Class: Quantifier}

Definition: an operator that specifies for which values of a variable a proposition is true.

\section{Member: forAll}

Definition: a logical quantifier of a proposition (within an expression) asserting that the proposition is true for all members of a class of objects.

\subsubsection{Primitive Class: Timepoint}

Definition: the exact point in time at which something may happen.

\subsubsection{Primitive Class: Identifier}

Definition: a word, symbol or phrase use to provide the identity of the optimization element bearing it.

\section{Conclusions}

This document proposes a specification for an optimization metamodel, which is intended to be used as a common reference for optimization problem definitions. The proposed specification provides an alternative technology to replace the current, multiple APIs implemented in commercial optimization tools. It also is an easy-to-use solution technique because it does not require the advanced knowledge and skills commonly needed to use those tools.

The main idea behind the work is to use the optimization metamodel as part of a new standard. This new standard has the potential to provide 1) a catalyst for a new or improved uses of optimization tools in manufacturing and 2) a component of emerging CPS architectures and model-based engineering activities. We invite the participation of others from the optimization-modeling-tools industry and experts from the standardization organizations to improve the semantic precision and make this potential a reality.

As it is today, the specification only uses the vocabulary of OPL; but, we intend to integrate other optimization-modeling-language vocabularies in the near future.

\section{Disclaimer}

Certain products or services are identified in the paper to foster understanding. Such identification does not imply recommendation or endorsement by the National Institute of Standards and Technology, nor does it imply that the products or services identified are necessarily the best available for the purpose.

\section{References}

[1] Object Management Group. 2011. OMG Meta Object Facility (MOF) Core Specification. Needham: Object Management Group.

[2] Object Management Group. 2011. "Unified Modeling Language (UML).", http://www.omg.org/spec/UML/2.4.1/. 
[3] Van Hentenryck P., Lustig I., Michel L., Puget J.F. 1999. The OPL “Optimization Programming Language.” The MIT Press, Cambridge, Massachusetts, London, England

[4] Fourer R., Gay D.M., Kernighan B.W. 1990. A Modeling Language for Mathematical Programming. Management Science, Vol. 36, No 5, pp. 519-554.

[5] Rosenthal R.E. 2015. GAMS -- A User's Guide. Washington DC: GAMS Development Corporation.

[6] Bussieck M.R., Meeraus A. 2004. "General algebraic modeling system (GAMS)." In modeling languages in mathematical optimization, pp. 137-157.

[7] Bisschop J., Entriken R. 1993. AIMMS "Advanced Interactive Multidimensional Modeling System (AIMMS): The modeling system.", Paragon Decision Technology.

[8] Jupyter Project. 2015. Jupyter Notebook. https://jupyter.org/.

[9] W3C. 2012. "OWL 2 Web Ontology Language.", http://www.w3.org/TR/owl2-primer/.

[10] World Wide Web Consortium. 2012. "OWL 2 Web Ontology Language Structural Specification and FunctionalStyle Syntax (Second Edition).", http://www.w3.org/TR/owl2-syntax/.

[11] Denno P. O., Kim D.B., to be published. "Integrating views of properties in models of unit manufacturing processes." International Journal of Computers in Manufacturing.

[12] NIST Modeling Methodology for Smart Manufacturing Systems. 2015, https://github.com/usnistgov/modelmeth/tree/master/models/optimization. 